\title{
GEOMETRIC TRANSFER AND THE HOMOTOPY TYPE OF THE AUTOMORPHISM GROUPS OF A MANIFOLD \\ BY
}

\author{
D. BURGHELEA AND R. LASHOF ${ }^{1}$
}

\begin{abstract}
Lifting concordances (pseudo-isotopies) in a smooth fibre bundle gives a transfer of stable concordance groups. Properties of the transfer are proved and exploited to obtain the homotopy structure of the group of diffeomorphisms or homeomorphisms of a manifold in a stable range.
\end{abstract}

Introduction. For $M$ a compact manifold with or without boundary in one of the categories Diff, PL or Top we denote by $A(M)$ the semisimplicial (ss) group of automorphisms of $M$ (i.e. the group of diffeomorphisms, PL homeomorphisms or homeomorphisms, resp.). In this paper we describe (in the stability range and localized away from two) the homotopy structure of $A(M)$. We do this in terms of $\tilde{A}(M)$, the ss group of block automorphisms (whose homotopy structure is a sophisticated formulation of surgery theory [ABK]) and some new homotopy functors $\delta_{ \pm}$(Theorem C).

The functors $\delta$ and $\delta \mathscr{U}$ here considered have already been studied in the PL category by Hatcher $[\mathbf{H}]$ from a different point of view. The present geometric description is appropriate to the description of the homotopy structure of $A(M)$ and enables us to produce a natural map $\beta: \mathcal{H}(X) \rightarrow \delta(X)$ from the associative monoid of simple homotopy equivalence of $X$ to $\delta(X)$ which will be important for our study.

Besides the geometric topology results [BLR] two ideas partially developed in [BL] are basic. The first is the "transfer map" and stability for concordances, described in the PL context by Hatcher and in the differentiable context in [BL]. The second is the splitting of a $Z\left(\frac{1}{2}\right)$ weak commutative (topological) group with involution as a product up to homotopy of two pieces, the symmetric, respectively antisymmetric parts. Applied to our situation those parts manifest very different behavior.

The results of this paper have been sketched by the first author in the survey paper [B]. There is an overlap between some (but not all) results of $\S A$ and results of Hatcher $\left[\mathbf{H}_{2}\right]$, published in the same volume as $[\mathbf{B}]$.

The results of this paper are stated in the next section (statement of results) and the proofs are given in subsequent sections. We continue this section with a description of the notations used.

Received by the editors November 8, 1979 and, in revised form, July 3, 1980.

1980 Mathematics Subject Classification. Primary 58D99, 58D10, 57E05; Secondary 55F10.

${ }^{1}$ Both authors were partially supported by the National Science Foundation. 
$\underline{A}(M ; K)$, resp. $\tilde{A}(M ; K)$, will denote the ss group of automorphisms, resp. block automorphisms, of $M$ which are the identity on $K \subset \partial M$. A typical $k$-simplex is essentially an automorphism $h: \Delta(k) \times M \rightarrow \Delta(k) \times M$ which commutes with projection on $\Delta(k)$, resp. is face preserving, and which restricts to the identity on $\Delta(k) \times K$ (see [BLR, Appendix I], for precise descriptions). To simplify the notation we write $\underline{A}(M)$, resp. $\tilde{A}(M)$ for $\underline{A}(M ; \varnothing)$, resp. $\tilde{A}(M ; \varnothing)$, and $A(M)$, $\tilde{A}(M), C(M), \tilde{C}(M)$ for $\underline{A}(M ; \partial \bar{M}), \tilde{A}(M ; \partial M), \underline{A}(M \times I ; \bar{\partial} M \times I \cup M \times(0))$, $\tilde{A}(M \times I ; \partial M \times I \cup M \times(0))$ resp. Since $\tilde{C}(M)$ is contractible, $\tilde{C}(M) / C(M)$ is homotopy equivalent to $B C(M)$, the classifying space of $C(M)$.

For a manifold with base point $\left(V, v_{0}\right)$, denote by $\operatorname{Emb}(M, M \times V)$ the ss complex of proper embeddings of $M$ in $M \times V$, and by $\operatorname{Emb}(M, M \times V)$ the ss subcomplex of $\operatorname{Emb}(M, M \times V)$ consisting of those proper embeddings which agree with the embedding $h_{0}, h_{0}(m)=\left(m, v_{0}\right)$, on $\partial M$. Also denote by $C \operatorname{Emb}(M, V)$ the ss complex of proper embeddings of $I \times M$ in $I \times M \times V$ which agree with id ${ }_{I} \times h_{0}$ on $I \times \partial M \cup(0) \times M$. (Recall that a proper embedding $h: V \rightarrow W$ is an embedding with $h^{-1}(\partial W)=\partial V$ and $h$ is transverse to $\partial W$.)

The covering map $\pi: M \times R \rightarrow M \times S^{1}$ induces $\pi: \operatorname{Emb}^{2}\left(M, M \times S^{1}\right) \rightarrow$ $\operatorname{Emb}(M, M \times R)$, resp. $C \pi: C \operatorname{Emb}\left(M, M \times S^{1}\right) \rightarrow C \operatorname{Emb}(M, M \times R)$, where $\mathrm{Emb}^{2}\left(M, M \times S^{1}\right)$ is the union of those connected components which when composed with the projection on $S^{1}$ are homotopy trivial. We write $\Re(M)$, resp. $C \Re(M)$, for the homotopy theoretic fibre of $\pi$, resp. $C \pi$. The spaces $\Re(M)$ and $C \Re(M)$ appear as factors in the direct product decomposition of $A\left(M \times S^{1}\right)$ and $C\left(M \times S^{1}\right)$ (see [BLR, Chapter VI]).

Denote by $\mathcal{F}(X)$ the ss associative monoid of simple homotopy equivalences of the compact polyhedra $X$, and for a manifold $M$ we let $\mathcal{H}(M)$ be the submonoid of $\mathcal{H}(M)$ of those simple homotopy equivalences of $M$ which restrict to the identity on $\partial M$.

Let $\mathbf{P}$ denote the category of finite polyhedra (spaces homeomorphic to the finite simplicial complex or CW complex) and continuous maps. If $f: X \rightarrow Y$ is a morphism in $\mathbf{P}$, we say it is a fibration in $\mathbf{P}$ if $f$ is a locally trivial bundle with fibre a compact manifold. It will be a Diff-bundle, PL-bundle, Top-bundle if the fibre $F$ is a manifold in the corresponding category with the group $\underline{\operatorname{Diff}}(F), \underline{\operatorname{PL}}(F), \underline{\operatorname{Top}}(F)$ resp.

For the ss Kan complex (or topological space) $X$, resp. the ss (or continuous) map $f: X \rightarrow Y$, we denote by $[X]_{k}$, resp. $[f]_{k}$, the $k$ th Postnikov term, and if $X$ is a nilpotent space (i.e., $\pi_{1}(X)$ is nilpotent and acts nilpotently on $\left.\pi_{m}(X)\right)$ denote by $X_{\text {odd }}$ the localization of $X$ away from two. The homotopy groups of $X_{\text {odd }}$ are $Z\left(\frac{1}{2}\right)$ modules.

We denote by $B$ the "classifying space" functor, $B G$ being the classifying space of a ss group (or associative monoid or loop space) $G$ and $B \theta$ the induced map on classifying spaces of a homomorphism $\theta: G \rightarrow G^{\prime}$.

Given $f, g: X \rightarrow Y$ we say that $f$ and $g$ are visibly homotopic if $[f]_{k}$ and $[g]_{k}$ are homotopic for any $k$; and we write $X \sim Y$ to denote $X$ homotopy equivalent to $Y$, $f \sim g$ to denote $f$ homotopic to $g$, resp. $X \stackrel{k}{\sim} Y$ and $f \stackrel{k}{\sim} g$ to denote $[X]_{k} \sim[Y]_{k}$ and $[f]_{k} \sim[g]_{k}$. 
Denote by $\stackrel{\infty}{\Omega}$ the category of infinite loop spaces and infinite loop space maps, $\stackrel{\infty}{\Omega}^{h}$, resp. $\stackrel{\infty}{\Omega}^{\text {h }}$, the homotopy category, resp. visible homotopy category of $\stackrel{\infty}{\Omega}$ whose objects are the objects of $\stackrel{\infty}{\Omega}$ and whose morphisms are homotopy classes, resp. visible homotopy classes of morphisms of $\stackrel{\infty}{\Omega}$.

If $\mathcal{S}$ is an object of $\stackrel{\infty}{\Omega}$ denote by $\hat{\mathcal{S}}$ the connected $\Omega$-spectrum associated with $\mathcal{S}$ whose components $\hat{\mathscr{S}}_{i}$ are given by

$$
\hat{\delta}_{i}= \begin{cases}* & \text { for } i<0 \\ \mathcal{E} & \text { for } i=0 \\ B^{i} \mathcal{S} & \text { for } i>0\end{cases}
$$

There will be more notations of local interest which will be indicated in the appropriate places.

Wherever the arguments require specification to the category Diff, PL or Top we will handle only the Diff and the Top case. The PL case is entirely similar to the Top case; it can be handled similarly (with more care on the geometric constructions to make sure we remain inside PL category) but it also follows from the last one by the "Comparation theorems" of the geometric categories.

Statement of results. The functors we describe in this paper are homotopy functors on $\mathbf{P}$ with values in $\Omega^{\text {vh }}$. They will be strongly connected. Since they are geometrically defined we will call them GH (geometric-homotopy) functors, although the descriptions below do not formalize their geometric origin.

Definition 1. A GH functor is a functor $\mathcal{L}: \mathbf{P} \rightarrow \stackrel{\infty}{\Omega}^{\text {vh }}$ satisfying:

(1) $f \sim g \Rightarrow \mathscr{L}(f)=\mathscr{L}(g)$.

(2) (a) If $f: X \rightarrow Y$ is $r$-connected, $\mathcal{L}(f): \mathcal{L}(X) \rightarrow \mathscr{L}(Y)$ is $(r-1)$-connected.

(b) If in the diagram

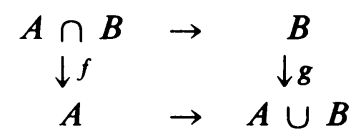

of inclusions in $\mathbf{P}$, the vertical arrows are $m$-connected, and the horizontal arrows are $n$-connected, $m, n \geqslant 2$, then the induced map Homotopy fibre $(\mathcal{L}(f)) \rightarrow$ Homotopy fibre $(\mathcal{L}(g))$ is $(m+n-2)$-connected.

(c) If $f: X \rightarrow Y$ is a rational equivalence (induces isomorphism for $\pi_{1}$ and for $\left.\pi_{i}() \otimes Q\right), \mathcal{L}(f): \mathcal{L}(X) \rightarrow \mathcal{L}(Y)$ is a rational equivalence.

(3) There exists a natural morphism $g$ in $\stackrel{\infty}{\Omega}^{v h}, g(X): \mathcal{L}\left(X \times S^{1}\right) \rightarrow B \mathcal{L}(X)$.

Let Nil $\mathcal{L}(X)=\operatorname{fibre}\left(g(X) \times \mathcal{L}\left(p_{X}\right)\right)$ : $\mathcal{L}\left(X \times S^{1}\right) \rightarrow B \mathscr{L}(X) \times \mathcal{L}(X)$. A GH functor $\mathcal{E}$ is said to satisfy WK (weak Kunneth property) if the fibration

$$
\text { Nil } \mathcal{L}(X) \rightarrow \mathcal{L}\left(X \times S^{1}\right) \rightarrow B \mathcal{L}(X) \times \mathcal{L}(X)
$$

is naturally trivial. In this case, Nil $\varrho$ defines a functor which satisfies (1) and (2) in Definition 1.

Definition 2. Let $\mathbb{Q}=$ Diff, PL or Top. The GH functor $\mathcal{L}=\mathcal{L}^{\mathbb{A}}$ has an $\mathbb{Q}$ transfer if for any $\mathcal{Q}$-bundle $\xi$ over $X$ (i.e. a locally trivial bundle with fibre $F$, a 
compact $\mathbb{Q}$ manifold, and structure group the $\mathscr{Q}$ automorphism group of $F$ ) there exists a transfer map $T(\varepsilon): \mathcal{L}(X) \rightarrow \mathcal{L}(E)$ which satisfies:

(1) If $E_{1} \stackrel{\xi_{1}}{\rightarrow} E_{2} \stackrel{\xi_{2}}{\rightarrow} X$ is a composition of $\mathcal{Q}$-bundles, $T\left(\xi_{2} \circ \xi_{1}\right)=T\left(\xi_{1}\right) \circ T\left(\xi_{2}\right)$.

(2) If

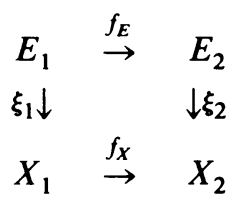

is a cartesian diagram with $\xi_{1}$ and $\xi_{2} Q$-bundles, then $T\left(\xi_{2}\right) \mathcal{L}\left(f_{X}\right)=\mathcal{L}\left(f_{E}\right) T\left(\xi_{1}\right)$.

(3) If $\varepsilon_{K}$ is the trivial $\mathbb{Q}$-bundle with fibre $K$ and $s: X \rightarrow X \times K$ is the cross-section $s(x)=\left(x, k_{0}\right)$, then $T\left(\varepsilon_{K}\right)=\chi(K) \mathcal{L}(s)$ (it makes sense to multiply a morphism in $\Omega^{\text {vh }}$ with an integer since $\Omega^{\text {vh }}$ is an additive category).

(4) $T$ is compatible with $g$; namely, if $\xi: E \rightarrow X$ is an $Q$-bundle

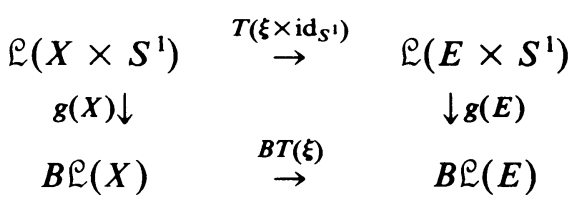

is commutative.

REMARKS. (a) Any generalized homology theory that vanishes in negative degrees is a $\mathrm{GH}$ functor with WK property and $\mathbb{Q}$ transfer.

In the definitions above we may replace $\stackrel{\infty}{\Omega}^{v h}$ with $\operatorname{Spec}^{v h}$, the visible homotopy category of spectra. The GH functors we will define are in fact 0 -components of such GH functors. For such functors (2) of Definition 1 implies that $\pi_{i}(\mathcal{L}(X))$, $i \leqslant 0$, depends only on $\pi_{1}(X)$.

Let $\tilde{K}_{\mathscr{Q}}(X)$ denote the reduced $K$-theory based on vector bundles, PL microbundles, microbundles for $\mathbb{Q}=$ Diff, PL, Top, respectively.

Definition 3. An $Q$ involution for the $\mathrm{GH}$ functor with $\mathcal{Q}$ transfer $\mathcal{L}=\mathcal{L}^{\mathbb{A}}$ means an involution $\tau^{\alpha}(X): \mathcal{L}(X) \rightarrow \mathcal{L}(X)$ for any $\alpha \in \tilde{K}_{Q}(X)$ so that:

(1) $\mathcal{L}(f):\left(\mathcal{L}(X), \tau^{f_{\alpha}^{*}}\right) \rightarrow\left(\mathcal{L}(Y), \tau^{\alpha}\right)$ for $\alpha \in \tilde{K}_{\alpha}(Y)$ is equivariant.

(2) If $\alpha \in \tilde{K}_{\mathscr{Q}}(X)$ and $p_{X}^{*} \alpha$ is the pull back of $\alpha$ to $X \times S^{1}, g(X)$ : $\left(\mathcal{L}\left(X \times S^{1}\right), \tau^{p_{x}^{*} \alpha}\right) \rightarrow\left(B \mathscr{L}(X), B \tau^{\alpha}\right)$ is equivariant.

An $Q$ involution $\tau$ on $\mathcal{L}$ produces functors $\mathcal{E}_{ \pm}: \mathbf{P}^{\mathbb{Q}} \rightarrow \stackrel{\infty}{\Omega^{v h}}$, where $\mathbf{P}^{\mathbb{Q}}$ denotes the category whose objects are pairs $(X, \alpha), X \in$ ob $\mathbf{P}$ and $\alpha \in \tilde{K}_{\alpha}(X)$; and morphisms $f:(X, \alpha) \rightarrow(Y, \beta)$, where $f: X \rightarrow Y$ is a map with $f_{\beta}^{*} \simeq \alpha$. The objects $\mathcal{E}_{ \pm}(X, \alpha)$ are obtained as the symmetric and antisymmetric parts of $\mathcal{L}(X)_{\text {odd }}$ under $\tau^{\alpha}$ (see $\S \mathrm{B}$ ).

Definition 4. The involutions $\tau^{\alpha}$ are called homotopy invariant (resp. rationally invariant) if for any $\alpha, \beta \in \tilde{K}_{\mathcal{Q}}(X)$ with $\alpha, \beta$ stably fibre homotopy equivalent (resp. $w_{1}(\alpha)=w_{1}(\beta)$ ) there exists an equivariant isomorphism $\theta(X, \alpha, \beta)$ : $\left(\mathscr{L}(X)_{\text {odd }}, \tau^{\alpha}\right) \rightarrow\left(\mathcal{L}(X)_{\text {odd }}, \tau^{\beta}\right)\left(\operatorname{resp} . \theta(X, \alpha, \beta):\left(\mathscr{L}(X)_{(0)}, \tau^{\alpha}\right) \rightarrow\left(\mathscr{L}(X)_{(0)}, \tau^{\beta}\right)\right) ; X_{(0)}$ will denote the localization to zero; i.e. $\pi_{i}\left(X_{(0)}\right)=\pi_{i}(X) \otimes Q$.

For the GH functors with $\mathcal{Q}$ involutions and transfer we will define, one can take $\theta(X, \alpha, \beta)=$ identity when $Q=$ PL or Top, and in fact we prove in these 
cases that $\tau^{\alpha}=\tau^{\beta}$ if $\alpha$ and $\beta$ are homotopy equivalent. We would like to have the same result for $Q=$ Diff, but our present argument breaks down in this case and we obtain the weaker result above. We would also like to have an $\mathbb{Q}$ involution well defined on our GH functors associated with any stable spherical fibration (because this is what the algebraic $K$-theory suggests) but this is unlikely to be produced by the methods of this paper.

An $\mathbb{Q}$ manifold $M^{n}$ determines a well-defined object in $\mathbf{P}^{\mathfrak{Q}}$; namely $\left(M^{n}, \alpha=\right.$ $T M)$ and it is well known that if $f:\left(M^{n}, \partial M\right) \rightarrow\left(N^{n}, \partial N\right)$ is a homotopy equivalence of compact manifolds with (possibly empty) boundaries, $f_{T N}^{*}$ is stably fibre homotopy equivalent to $T M$. For $\mathcal{L}$ a $\mathrm{GH}$ functor with homotopy invariant involution, $\varrho_{+}\left(M^{n}\right) \sim \mathcal{L}_{+}\left(N^{n}\right), \quad \varrho_{-}\left(M^{n}\right) \sim \mathcal{L}_{-}\left(N^{n}\right)$, where $\mathcal{L}_{ \pm}(M)=$ $\mathcal{L}_{ \pm}(M, T M)$, whenever $M^{n}, N^{n}$ are homotopy equivalent manifolds with boundary. The dimension (which is invariant under homotopy equivalence for compact manifolds with boundary) specifies a choice between $\mathcal{L}_{+}(M)$ and $\mathcal{L}_{-}(M)$; namely, $\mathcal{L}_{e(n)}(M)$, where $\varepsilon(n)=+$ if $n$ is odd and - if $n$ is even.

THEOREM A. (1) For any geometric category $Q$ there exists a GH functor $\mathcal{S}=\mathcal{S}^{\mathbb{Q}}$ with $W K, \mathbb{Q}$ transfer and $\mathbb{Q}$ involution.

(2) If $\mathbb{Q} \leqslant \mathscr{B}$, there exists a natural transformation $\mathfrak{S}^{\mathbb{Q}} \rightarrow \mathcal{S}^{\mathscr{B}}$, compatible with $W K, \mathbb{Q}$ transfer and homotopy invariant $\mathbb{Q}$ involution. Consequently, it induces the natural transformation $\mathcal{S}_{ \pm}^{\mathcal{Q}} \rightarrow \mathcal{S}_{ \pm}^{\mathscr{B}}$ on $\mathbf{P}^{\mathfrak{Q}}$. All these transformations are isomorphisms for $\mathbb{Q}=\mathrm{PL}, \mathscr{B}=$ Top.

(3) $\mathrm{Nil} \mathcal{S}^{\mathbb{Q}}=$ Nil $\mathcal{S}^{\mathscr{B}}$, Nil $\mathcal{S}_{ \pm}^{\mathbb{Q}} \simeq$ Nil $\mathcal{S}_{ \pm}^{\mathscr{B}}$.

(4) The homotopy theoretic fibre of $\varsigma^{\bar{Q}}(X) \rightarrow \varsigma^{\mathscr{B}}(X)$ defines a functor which is $a$ generalized homology theory given by the spectra $\hat{\delta}^{\mathbb{Q}}(*)$. In particular, $\hat{\mathcal{S}}^{\mathrm{PL}}(*)=$ $\hat{\hat{S}}^{\mathrm{Top}}(*)=*$.

$\pi_{i}\left(\right.$ Fibre $\left.\left(\mathcal{S}_{ \pm}^{\mathcal{Q}}(X, \alpha) \rightarrow \mathcal{S}_{ \pm}^{\mathscr{G}}(X, \alpha)\right)\right)$ does not depend on $\alpha$. Hence taking $\alpha$ trivial for all $X$, the homotopy theoretic fibre defines a homology theory given by the spectra $\hat{\mathcal{S}}_{ \pm}^{\mathbb{Q}}(*)=\hat{\mathscr{S}}_{ \pm}^{\mathbb{Q}}(*, \mathcal{E})$. In particular, $\hat{\mathcal{S}}_{ \pm}^{\mathrm{PL}}(*)=\hat{\mathscr{S}}_{ \pm}^{\mathrm{Top}}(*)=*$.

(5) There exists an increasing function $\omega(n)=\omega^{\mathbb{Q}}(n)$ called the "stability range" $(\omega(n) \rightarrow \infty)$, so that for any compact manifold $M$ :
(a) $\tilde{C}\left(M^{n}\right) / C\left(M^{n}\right) \stackrel{\omega(n)+1}{\sim} \delta\left(M^{n}\right)$,
(b) $\left(\bar{A}\left(M^{n}\right) / A\left(M^{n}\right)\right) \stackrel{\omega(n)+1}{\sim} \delta_{\mathrm{e}(n)}\left(M^{n}\right)$,
(c) $C \Re\left(M^{n}\right) \stackrel{\omega(n+1)}{\sim} \Omega$ Nil $\delta\left(M^{n}\right)$,
(d) $\Re\left(M^{n}\right)_{o d d} \stackrel{\omega(n+1)}{\sim} \Omega$ Nil $\delta_{\varepsilon(n)}\left(M^{n}\right)$, where $\varepsilon(n)=+$ if $n$ is odd and $\varepsilon(n)=-$ if $n$ is even.

Further the equivalences are natural with respect to inclusions $M^{n} \subset N^{n}$ and with respect to the category $Q$.

THEOREM B. (a) For any $X \in \mathrm{ob} \mathbf{P}$, there exists a well-defined homotopy class $\beta(X)=\beta^{\mathcal{Q}}(X): \underline{\mathcal{H}}(X) \rightarrow \mathcal{S}^{\mathbb{Q}}(X)$, natural with respect to category.

(b) If $f: X \rightarrow \bar{Y}$ is a simple homotopy equivalence and $f_{\#}: \mathcal{F}(X) \rightarrow \underline{\mathcal{H}}(Y)$ is the map (defined up to homotopy) induced by conjugation with $f$, then $\mathcal{S}(f) \beta(X) \sim$ $\beta(Y) f_{\#}$. 
(c) For any compact manifold $V$, the following diagram is commutative:

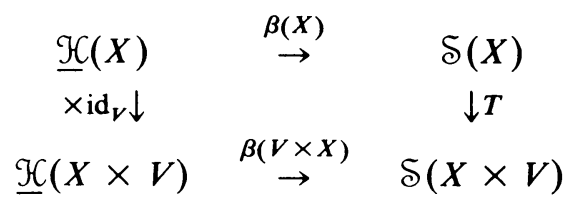

(d) If $\alpha, \alpha^{\prime} \in \tilde{K}(X)$ are stably fibre homotopy equivalent $\left(\right.$ resp. $\left.w_{1}(\alpha)=w_{1}\left(\alpha^{\prime}\right)\right)$ then

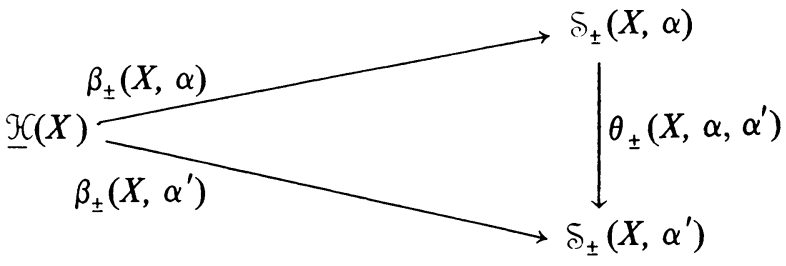

commutes, where $\beta_{ \pm}(X, \alpha)$ is the composition of $\beta(X)$ with the projection of $\mathcal{S}(X)$ into $\mathcal{S}_{ \pm}(X, \alpha)$ (resp. into $\mathcal{S}_{ \pm}(X, \alpha)$ localized at zero).

(e) $\pi_{0}(\mathcal{H}(X)) \rightarrow \pi_{0}(\mathcal{S}(X))$ is trivial.

REMARKs. (1) For $Q=\mathrm{PL}, \beta(X)$ is probably the same as Hatcher's map of $\mathcal{F}(X)$ to $\Omega W h^{\mathrm{PL}}(X)[\mathbf{H}]$.

(2) We would like to deloop $\beta(X)$, but the construction we use does not allow this.

(3) Actually $\delta(X)$ is a connected component of $\Omega W h^{\mathrm{PL}}(X)$ and our $\beta(X)$ has an extension from the monoid of all homotopy equivalences of $X$ to $\Omega W h^{\mathrm{PL}}(X)$ which at the $\pi_{0}$ level is exactly Whitehead torsion associated to a homotopy equivalence.

The map $\beta(X): \mathcal{H}(X) \rightarrow \delta(X)$ induces the principal fibration

$$
\Omega \delta(X) \rightarrow \xi(X) \rightarrow \underline{\mathcal{H}}(X)
$$

from the space of paths fibration over $\delta(X)$.

The maps $\beta_{ \pm}(X, \alpha): \underline{F}(X) \rightarrow \delta_{ \pm}(X, \alpha)$ induce the principal fibrations

$$
(I, \alpha)_{ \pm} \quad \Omega \mathcal{S}_{ \pm}(X, \alpha) \rightarrow \xi_{I}(X, \alpha) \rightarrow \underline{\mathcal{H}}(X),
$$

from the path space fibration over $\delta_{ \pm}(X, \alpha)$.

It is clear that $(I)$ fibrewise localized away from two is the Whitney sum of $(I, \alpha)_{+}$and $(I, \alpha)_{-}$. On the other hand, $(I, \alpha)_{ \pm}$fibrewise localized at zero depends only on $w_{1}(\alpha)$.

In particular, the homotopy type of a compact manifold with boundary $\left(M^{n}, \partial M^{n}\right)$ determines a particular choice of $(I, T M)_{ \pm},+$if $n$ is odd, - if $n$ is even. Thus we get a principal fibration $h(M): \Omega \delta_{e(n)}(M) \rightarrow \xi_{e(n)}(M) \rightarrow \mathscr{H}_{(}(M)$, which is a homotopy theoretic invariant of compact manifolds with boundary.

The geometric $Q$ structure on $M^{n}$ produces an explicit map $\tilde{A}(M) \stackrel{i(M)}{\rightarrow} \mathscr{H}(M)$, whose understanding is the aim of surgery theory (and this aim is theoretically achieved). Theorem $\mathrm{C}$ below basically says that away from the prime two and in 
the stable range $A\left(M^{n}\right)$ is the homotopy pull back of $h(M): \xi_{e(n)} \rightarrow \mathscr{H C}(M)$, a homotopy theoretic invariant, by $i(M): \tilde{A}(M) \rightarrow \underline{F}(M)$, the surgery theory map.

TheOREM C. If one denotes by $I I\left(M^{n}\right)$, the pull back by $i(M): \tilde{A}(M) \rightarrow \underline{H}(M)$ of the principal fibration $h(M): \xi_{e(n)} \rightarrow \mathcal{H}(M)$, this is $\omega(n)+1$ isomorphic to the fibrewise localization away from two of the fibration $\Omega \tilde{A}(M) / A(M) \rightarrow A(M) \rightarrow$ $\tilde{A}(M)$.

Recall that two principal fibrations induced from the maps $f: X \rightarrow B G_{1}$ and $g$ : $X \rightarrow B G_{2}$ are $k$-isomorphic if there exists a $k$-homotopy equivalence $\theta:\left[B G_{1}\right]_{k} \rightarrow$ $\left[B G_{2}\right]_{k}$ so that $\theta \circ[f]_{k} \sim[g]_{k}$.

Corollary D. $\Omega(\underline{\mathcal{H}}(M) / A(M))_{o d d} \sim^{\omega(n)} \Omega(\mathcal{F}(M) / \tilde{A}(M))_{o d d} \times \Omega S_{e(n)}(M)$.

The isomorphism of the homotopy groups of $(\underline{H}(M) / A(M))_{\text {odd }}$ and $(\underline{F}(M) / \tilde{A}(M))_{\text {odd }} \times \Omega S_{e(n)}(M)$ has been independently noticed by Hsiang and Jahren $[\mathbf{H J}]$.

COROLlaRY E. If $M^{n}$ is an $\mathbb{Q}$ manifold so that there exists a map $p: M \rightarrow \partial M$ with $M \stackrel{p}{\rightarrow} \partial M \stackrel{i}{\rightarrow} M$ homotopic to the identity, then $I I\left(M^{n}\right)$ is trivial over $[\tilde{A}(M)]_{\omega(n)+1}$ and hence

$$
A\left(M^{n}\right)_{\text {odd }} \stackrel{\omega(n)+1}{\sim} \Omega S_{e(n)}(M) \times \tilde{A}(M)_{o d d} .
$$

CoROllary F. (1) If $M^{n} \subset E^{n}$ is an r-connected codimension 0 submanifold, then (*) holds for the kth Postnikov term, where

(a) $k=\inf (\omega(n), 2 r-2)$ in the Diff category,

(b) $k=\inf (\omega(n), 2 r-2, r+3)$ in the PL or Top category.

(2) If $\left(M^{n}, \partial M\right)$ has the homotopy type of $\left(N^{n-1} \times S^{1}, \partial\left(N \times S^{1}\right)\right)$, then $\Omega(I I(M))$ is trivial over the $\omega(n)$ Postnikov term and consequently $\Omega A(M)_{\text {odd }}$ $\stackrel{\omega(n)}{\sim} \Omega \tilde{A}(M)_{\text {odd }} \times \Omega^{2} S_{e(n)}(M)$.

(3) If $M^{n}$ is a $K(\pi, 1)$ manifold then the conclusion of (2) holds.

Note. The results discussed above provide exact computations of the homotopy groups tensored by the rationals of $\operatorname{Diff}(M), \operatorname{Homeo}(M)$ and $\operatorname{PL}(M)$ in stability range in few concrete cases; see $\left[\mathbf{B}_{3}\right]$ and its references. All manifolds for which such computations have been done enjoy the following properties:

(i) $\pi_{i}(H(M)) \otimes Q$ can be computed at least in stability range;

(ii) $\pi_{i}(\overparen{\operatorname{Diff}}(M)) \otimes Q=\pi_{i}(\widetilde{\mathrm{Homeo}}(M)) \otimes Q$ can be computed by the means of surgery theory plus the computations in (i);

(iii) the fibration $(I)$ or at least the fibration obtained by applying the loop space functor $\Omega$ to $(I)$ is trivial; Corollaries $\mathrm{E}$ and $\mathrm{F}$ provide enough examples;

(iv) $M$ is either 1-connected or $K(\pi, 1)$ in stability range, in which case $\pi_{i}\left(\mathcal{S}_{e(n)}(M)\right) \otimes Q$ is reduced via the Waldhausen theory [W] to the algebraic $K$-theory of the space $X=M$.

Upper bound estimates (and exact computations in a few particular cases) of the algebraic $K$-theory of $X$ tensored by the rationals have been obtained for all 1-connected spaces by Burghelea and Dwyer, Hsiang, Staffeldt (see $\left[\mathbf{B}_{3}\right]$ and the 
forthcoming paper of the above-mentioned authors); if $M$ is a $K(\pi, 1)$, the algebraic $K$-theory of $M$ identifies with the algebraic $K$-theory of the ring $Z(\pi)$.

A. Transfer. If $M=N \times I$ and $h, g \in \underline{A}(N \times I ; N \times \partial I)$, juxtaposition along $I, h \# g$, is defined as follows: $h \# g \mid N \times\left[0, \frac{1}{2}\right]=\left(1 \times \nu^{-1}\right) \circ h \circ(1 \times \nu)$ and $h \# g \mid N \times\left[\frac{1}{2}, 1\right]=\left(1 \times \mu^{-1}\right) \circ g \circ(1 \times \mu)$, where $\nu:\left[0, \frac{1}{2}\right] \rightarrow[0,1]$ and $\mu:\left[\frac{1}{2}, 1\right] \rightarrow$ $[0,1]$ are given by $\nu(t)=2 t$ and $\mu(t)=1-2 t$.

If $M=N \times I^{r}$ we can perform juxtapositions between

$$
h, g \in \underline{A}\left(N \times I^{r} ; N \times \partial I^{r}\right)
$$

in essentially $r$ ways by picking up one of the factors of $I^{r}$. We observe that composition and the various juxtapositions are homomorphisms with respect to each other. Since juxtaposition is a loop space type multiplication it has a classifying space. More generally using the "little cubes" recognition principle [M], it is easy to see that composition deloops $r$ times to $B^{r} \underline{A}\left(N \times I^{r} ; N \times \partial I^{r}\right)$.

Juxtaposition may also be defined for $h, g \in C(M)$ where $M=N \times I$ or, more generally, $N \times I^{r}$. Again composition deloops $r$ times to $B^{r} C\left(N \times I^{r}\right)$ and thus $C\left(N \times I^{r}\right)$ has an $r+1$ delooping. It follows that composition is homotopic to juxtaposition. Juxtaposition may also be defined for $\tilde{A}\left(N \times I^{r} ; N \times \partial I^{r}\right)$ and $\tilde{C}\left(N \times I^{r}\right)$ with the corresponding properties holding.

For $h \in C(M), h: M \times I \rightarrow M \times I$, we have $h_{1}: M \rightarrow M$; i.e. $h(x, 1)=$ $\left(h_{1}(x), 1\right)$. Define $\tau: C(M) \rightarrow C(M)$ by

$$
\tau(h)=h^{R} \circ\left(h_{1}^{-1} \times 1\right), \quad h^{R}=(1 \times \lambda) \circ h \circ(1 \times \lambda), \quad \lambda(t)=1-t .
$$

Note that $\tau(h)_{1}=h_{1}^{-1}$, so that $\tau$ is an involution. Further $\tau$ is a homomorphism with respect to juxtaposition in $C\left(N \times I^{r}\right)$ and so deloops $r$ times to an involution on $B^{r} C\left(N \times I^{r}\right)$, denoted abusively also by $\tau$.

The construction " $j$ ". Let $M^{n}=\left(M ; M_{i}\right), i=1, \ldots, r$, be an $n$ manifold with corners; i.e. the $M_{i}$ are the $n-1$ faces of $M$. Then $M \times I=\left(M \times I ; M \times 0, M_{i}\right.$ $\times I, M \times 1)$ is again a manifold with corners. For any face $M_{j}$ of $M$, let $\tilde{M}^{j}=M \cup M_{j} \times[0,1]$ identified along $M_{j}=M_{j} \times(0)$. Then $\tilde{M}^{j}=\left(\tilde{M}^{j} ; \tilde{M}_{i}^{j}\right)$, where $\tilde{M}_{i}^{j}=M_{i} \cup\left(M_{i} \cap M_{j}\right) \times[0,1]$ if $i \neq j$, and $\tilde{M}_{j}^{j}=M_{j} \times(1)$. See Figure 1 .

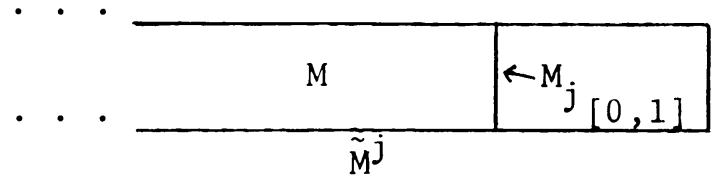

\section{Figure 1}

Denote by $\underline{A}^{c}(M)$ the automorphisms of $M$ as a manifold with corners; i.e. the subgroup of automorphisms of $M$ which preserve faces. Let $\partial_{j} M=\cup_{i<j} M_{i}$, and let $\underline{A}^{c}\left(M ; \partial_{j} M\right)$ be the subgroup of $\underline{A}^{c}(M)$ of automorphisms which are the identity on $\partial_{j} M$. The restriction map $\rho_{j}: \underline{A}^{c}\left(M ; \partial_{j-1} M\right) \rightarrow \underline{A}^{c}\left(M_{j} ; M_{j} \cap \partial_{j-1} M\right)$ is a fibration with fibre $\underline{A}^{c}\left(M ; \partial_{j} M\right)$. 
Similarly we have the restriction map

$$
\begin{aligned}
\rho_{j}: \underline{A}^{c}(M \times I ; M & \left.\times 0 \cup \partial_{j-1} M \times I\right) \\
& \rightarrow \underline{A}^{c}\left(M_{j} \times I ; M_{j} \times 0 \cup\left(M_{j} \cap \partial_{j-1} M\right) \times I\right)
\end{aligned}
$$

with fibre $\underline{A}^{c}\left(M \times I ; M \times 0 \cup \partial_{j} M \times I\right)$. We will define a homomorphism

$$
\beta_{j}: \underline{A}^{c}\left(M \times I ; M \times 0 \cup \partial_{j-1} M \times I\right) \rightarrow \underline{A}^{c}\left(M \times I ; M \times 0 \cup \partial_{j} M \times I\right)
$$

which is a left (homotopy) inverse to the inclusion $i_{j}$ of the fibre.

First note that since $M_{j}$ has a collar neighborhood in $M, \tilde{M}^{j}$ is isomorphic to $M$, and we can identify

$$
\underline{A}^{c}\left(\tilde{M}^{j} \times I ; \tilde{M}^{j} \times 0 \cup \partial_{j} \tilde{M}^{j} \times I\right) \text { with } \underline{A}\left(M \times I ; M \times 0 \cup \partial_{j} M \times I\right) .
$$

Given $h \in \underline{A}^{c}\left(M \times I ; M \times 0 \cup \partial_{j-1} M \times I\right)$, define

$$
\beta_{j}(h) \in \underline{A}^{c}\left(\tilde{M}^{j} \times I ; \tilde{M}^{j} \times 0 \cup \partial_{j} \tilde{M}^{j} \times I\right) \text { by } \beta_{j}(h) \mid(M \times I)=h,
$$

and taking polar coordinates $(r, \theta)$ in $[0,1] \times I$ (see Figure 2) $\beta_{j}(h)$ in $(x, r, \theta) \in$ $M_{j} \times[0,1] \times I$ is

$$
\beta_{j}(h)(x, r, \theta)= \begin{cases}\left(h_{1}(x, 1-r), 1-h_{2}(x, 1-r), \theta\right), & r \leqslant 1, \\ (x, r, \theta), & r \geqslant 1,\end{cases}
$$

where $h(x, t)=\left(h_{1}(x, t), h_{2}(x, t)\right)$. I.e., $\beta_{j}(h) \mid\left(M_{j} \times[0,1] \times I\right)$ is obtained by rotating the concordance $h \mid M_{j} \times I$ around $M_{j} \times(0) \times(1)$ in $M_{j} \times[0,1] \times I$. Note that if $h$ is in $i_{j} \underline{A}^{c}\left(M \times I ; M \times 0 \cup \partial_{j} M \times I\right), h \mid M_{j} \times I=$ identity and $\beta_{j}(h) \mid M_{j} \times$ $[0,1] \times I$ is the identity. Hence $\beta_{j}(h)$ is obtained from $h$ by "thickening the collar"; i.e. $\beta_{j} \circ i_{j} \sim$ identity.

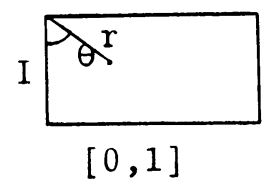

FIGURE 2

Now $C(M)=\underline{A}^{c}(M \times I ; M \times 0 \cup \partial M \times I)$. Also note that $\partial M=\partial_{r} M$. Thus starting with $\underline{A}^{c}(M \times I ; M \times 0)$ or any $\underline{A}^{c}\left(M \times I ; M \times 0 \cup \partial_{j} M \times I\right)$ we can construct a homomorphism $\sigma_{j}: \underline{A}^{c}\left(M \times I ; M \times 0 \cup \partial_{j} M \times I\right) \rightarrow C(M), j>0$ $\left(\partial_{0} M=\varnothing\right)$, which is a left homotopy inverse of the inclusion. Similarly, one may construct $\tilde{\sigma}_{j}: \tilde{A}^{c}\left(M \times I ; M \times 0 \cup \partial_{j} M \times I\right) \rightarrow \tilde{C}(M)$; the restriction of $\tilde{\sigma}_{j}$ being $\sigma_{j}$. This defines a map of pairs $\tilde{\sigma}_{j}:\left(\tilde{A}^{c}(), \underline{A}^{c}()\right) \rightarrow(\tilde{C}(M), C(M))$ and induces a map of quotients $\bar{\sigma}_{j}: \tilde{A}^{c}() / \underline{A}^{c}() \rightarrow \tilde{C}(M) / \bar{C}(M)$.

We note that the involutions $\tilde{\tau}, \tau$ are actually defined on $\tilde{A}^{c}(), \underline{A}^{c}()$, and they commute with the inclusions $\tilde{i_{j}}, i_{j}$. Unfortunately, they do not commute with the $\beta_{j}$ 's.

Finally we note that $\tilde{\sigma}_{j}$ and $\sigma_{j}$ are unaffected by the ordering of the faces of $M$ so long as $\partial_{j} M$ remains the union of the same collection of faces. This follows from the fact that, up to deformation retract, we may assume $\underline{A}^{c}(M)$ (and $\underline{A}^{c}(M \times I)$ ) consists of automorphisms which are products with $\mathrm{id}_{C^{k}}$ along a neighborhood of 
any $(n-k)$ edge in $M$, where $C^{k}$ is a $k$-corner. For the same reason $C(M)$ is independent of the corners in $M$; i.e. we get the same group if we round all the corners.

Now let $\xi: E \rightarrow M$ be a locally trivial bundle with fiber $F$ a compact manifold; and let $\partial_{-} E=\xi^{-1}(\partial M)$ and $\partial_{+} E=\overline{\partial E-\partial_{-} E}$. We note that even if $M$ has no corners, if $\partial F \neq \varnothing$ then $\partial_{+} E \neq \varnothing$ and $E$ is a manifold with corners. Further, it will be necessary to consider the case where $F$ has corners and the group of the bundle preserves the faces of $F$. Then $\partial_{+} E$ will be a union of faces.

Let $\underline{A}_{s}\left(E \times I ; \quad E \times 0 \cup \partial_{-} E \times I\right)$ be the subgroup of those $h \in$ $\underline{A}^{c}\left(E \times I, E \times 0 \cup \partial_{-} E \times I\right)$ which are bundle automorphisms over $\bar{h} \in C(M)$. The homomorphism $h \rightarrow \bar{h}$ defines a fibration

$$
\pi(\xi): \underline{A}_{s}\left(E \times I ; E \times 0 \cup \partial_{-} E \times I\right) \rightarrow C(M)
$$

with fibre consisting of all bundle automorphisms over $\mathrm{id}_{M \times I}$ which are fixed over $M \times 0 \cup \partial M \times I$. That $\pi(\xi)$ is surjective and a fibration follows from the covering homotopy property of $\xi$. Similarly, we get a fibration

$$
\tilde{\pi}(\xi): \underline{\tilde{A}}_{s}\left(E \times I ; E \times 0 \cup \partial_{-} E \times I\right) \rightarrow \tilde{C}(M)
$$

with exactly the same fibre and hence an isomorphism

$$
\bar{\pi}_{s}: \underline{A}_{s}() / \underline{A}_{s}() \rightarrow \tilde{C}(M) / C(M) .
$$

But in any case the fibre of $\pi(\xi)$ (or $\tilde{\pi}(\xi))$ is obviously contractible, so that $\pi(\xi)$ and $\tilde{\pi}(\xi)$ are homotopy equivalences.

The involutions $\tilde{\tau}, \tau$ restrict to $\tilde{A}_{s}(), A_{s}($ ) resp., and $\tilde{\pi}(\xi), \pi(\xi)$ commute with $\tilde{\tau}, \tau$ resp. If $M=N \times I^{r}$, then $E=\bar{E}_{N} \times \bar{I}^{r}, E_{N}=E \mid N \times(0)$, and $\underline{A}_{s}(), \underline{A}_{s}(\mathrm{)})$ have an $r$ loop space structure which is preserved by $\tilde{\pi}(\xi), \pi(\xi)$ resp.

The transfer. Given $\xi: E \rightarrow M$ as above, define the transfer $T=T(\xi): C(M) \rightarrow$ $C(E)$ as the composition $T=\sigma_{-}{ }^{\circ} \pi^{-1}: C(M) \rightarrow A_{s}\left(E \times I, E \times 0 \cup \partial_{-} E \times I\right)$ $\rightarrow C(E)$. Define $\tilde{T}: \tilde{C}(M) \rightarrow \tilde{C}(E)$ and $\bar{T}: \tilde{C}(M) / \vec{C}(M) \rightarrow \tilde{C}(E) / C(E)$ similarly. $T$ and $\tilde{T}$ are defined up to homotopy, whereas $\bar{T}$ is uniquely defined.

It will be possible to choose a specific right inverse to $\pi(\xi)$ as soon as we introduce a "parallel transport" on $\xi$. A parallel transport $\Gamma$ in a Diff-bundle $\xi$ associates to any piecewise differentiable path $\lambda:[a, b] \rightarrow M$ a diffeomorphism $\Gamma(\lambda)=\lambda_{*}: \xi^{-1}(\lambda(a)) \rightarrow \xi^{-1}(\lambda(b))$ with the usual properties. For any concordance $k: M \times I \rightarrow M \times I, k(x, t)=\left(k_{1}(x, t), k_{2}(x, t)\right)$, we define $\gamma(k): E \times I \rightarrow E \times I$ by $\gamma(k)(z, t)=\left(\left(k_{1, x}^{t}\right)_{*} z, k_{2}(x, t)\right)$, where $k_{1, x}^{t}:[0, t] \rightarrow M$ is the path $k_{1, x}^{t}(s)=$ $k_{1}(x, s)$, and $x=\xi(z)$.

If $\xi$ has a Lie structure group $G$, then we may obtain a parallel transport in the usual way by choosing a connection in the associated principal bundle. Then for any $k \in C(M), \gamma(k)$ will be a $G$ bundle isomorphism.

For an arbitrary differentiable bundle with compact fibre one may always define a parallel transport by choosing a Riemannian metric on $E$ respecting $\partial_{+} E$. Given a path $\lambda:[a, b] \rightarrow M$ and the tangent vector $\lambda^{\prime}(t)$ to $\lambda(t)$, we may lift $\lambda^{\prime}(t)$ to any point of $\xi^{-1}(\lambda(t))$ by taking the unique vector orthogonal to the fibre which projects onto $\lambda^{\prime}(t)$. The integral curves of this vector field over $\lambda$ define a parallel transport. 
For a topological bundle we define parallel transport over arbitrary paths in $M$ as follows:

Let $p_{0}: M^{I} \rightarrow M, p_{0}(w)=w(0)$. Let $P_{0}=p_{0}^{*}(\xi), P_{0}=\left\{(w, z) \in M^{I} \times E \mid w(0)=\right.$ $\xi(z)\}$. Consider the homotopy $p: M^{I} \times I \rightarrow M, p(w, t)=w(t)$. By the CHP there is a bundle map $\phi: P_{0} \times I \rightarrow E$ covering $p_{0}$. For $\lambda:[a, b] \rightarrow M$ define $\lambda_{*}: \xi^{-1}(\lambda(a))$ $\rightarrow \xi^{-1}(\lambda(b))$ by $\lambda_{*}(z)=\phi((\bar{\lambda}, z), 1)$, where $\bar{\lambda}: I \rightarrow M, \bar{\lambda}(t)=\lambda(a+t(b-a))$. Note that if $\xi$ has the topological structure group $G$, then since $\phi$ is a $G$ bundle map, $\lambda_{*}$ is a $G$ bundle isomorphism and $\gamma(k)$ will be a $G$ bundle isomorphism for $k \in C(M)$, $\gamma(k)$ defined as above.

Now if $M=N \times I^{r}$ one may always choose parallel transport in $E=E_{N} \times I^{r}$ by starting with a parallel transport $\Gamma_{N}$ in $E_{N}$ and defining $\Gamma(\lambda)$ for $\lambda:[a, b] \rightarrow N$ $\times I^{r}, \lambda(t)=\left(\lambda_{1}(t), \lambda_{2}(t)\right)$, by $\Gamma(\lambda)=\Gamma_{N}\left(\lambda_{1}\right): \xi^{-1}(\lambda(a)) \cong \xi_{N}^{-1}\left(\lambda_{1}(a)\right) \rightarrow \xi_{N}^{-1}\left(\lambda_{1}(b)\right)$ $\cong \xi^{-1}(\lambda(b))$. Then $\gamma: C(M) \rightarrow A_{s}\left(E \times I, E \times 0 \cup \partial_{-} E \times I\right)$ will preserve the $r$ loop space structure. Since $\sigma_{-}: \underline{A}_{s}() \rightarrow C(E)$ clearly preserves the $r$ loop space structure, $T$ will preserve the $r$ loop space structure if we choose $T=\sigma_{-} \circ \gamma$. Thus $T$ deloops $r$ times. This delooping will be independent (up to homotopy) of the choice of $\Gamma$ since $\pi(\xi)$ is an $r$ loop space map and a homotopy equivalence.

From now on we will denote the transfers $T, \tilde{T}, \bar{T}$ indiscriminately by $T$ and similarly the involutions $\tau, \tilde{\tau}, \bar{\tau}$ by $\tau$, etc. The specific choice will be indicated by the context.

The suspension. If $\xi: M \times F \rightarrow M$ is the trivial bundle, then we can choose parallel transport so that $\gamma: C(M) \rightarrow \underline{A}_{s}\left(E \times I ; E \times 0 \cup \partial_{-} E \times I\right)$ is just product with id ${ }_{F}$. In particular, if $F=I$, then $T: C(M) \rightarrow C(M \times I)$ is easily seen to be equivalent to rotating the concordance $h: M \times I \rightarrow M \times I$ by $180^{\circ}$ in $M \times I \times$ I;

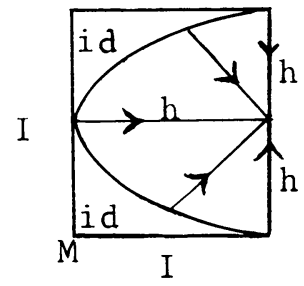

where the arrow indicates the direction from 0 to 1 . This is the Hatcher and Wagoner stability homomorphism $\Sigma: C(M) \rightarrow C(M \times I)$ (see [H] and [BL]). If $\xi$ : $M \times D^{k} \rightarrow M$ we denote $T(\xi)$ by $\Sigma^{k}$.

Properties of the transfer.

Proposition Al. If $\xi^{\prime \prime}: E^{\prime} \stackrel{\xi^{\prime}}{\rightarrow} E \stackrel{\xi}{\rightarrow} M$ is a composition of bundles, then $T\left(\xi^{\prime \prime}\right) \sim$ $T\left(\xi^{\prime}\right) \circ T(\xi)$.

Proof. Choosing parallel transport in $\xi^{\prime \prime}$ by composition, we have $\gamma^{\prime \prime}=\gamma^{\prime} \circ \gamma$. Then $T\left(\xi^{\prime \prime}\right)$ differs from $T\left(\xi^{\prime}\right) \circ T(\xi)$ only because of the ordering of the faces. The result follows.

REMARKS. (a) If $M=N \times I^{r}$, this is a homotopy of $r$ loop space homomorphisms.

(b) If $E=M \times D^{k}, \Sigma^{k} \sim(\Sigma)^{k}$. 
Proposition A2. Given $\xi: E \rightarrow M^{n}$ and $N^{n} \subset M^{n}$, then

$$
\begin{array}{ccc}
C(N) & \stackrel{i}{\rightarrow} & C(M) \\
T(\varepsilon \mid N) \downarrow & & \downarrow T(\varepsilon) \\
C(E \mid N) & \stackrel{i}{\rightarrow} & C(E)
\end{array}
$$

commutes. Similarly for $\tilde{C}$ and $\tilde{C} / C$.

Proof. This is immediate from the definition.

Proposition A3. Let $\xi: E^{n+k} \rightarrow M^{n}$ be the union of two subbundles $\xi_{i}: E_{i}^{n+k} \rightarrow$ $M^{n}, i=1,2$, intersecting in a common face, $\xi_{3}: E_{3}^{n+k-1} \rightarrow M^{n}$. Let $i_{3}: E_{3} \times I \rightarrow E$ be the inclusion of a collar neighborhood, and $i_{1}, i_{2}$ the inclusions of $E_{1}$ and $E_{2}$ in $E$. Then

$$
T(\xi)=i_{1} T\left(\xi_{1}\right) \cdot\left(i_{3} \Sigma T\left(\xi_{3}\right)\right)^{-1} \cdot i_{2} T\left(\xi_{2}\right)
$$

Proof. Diagrammatically we have:

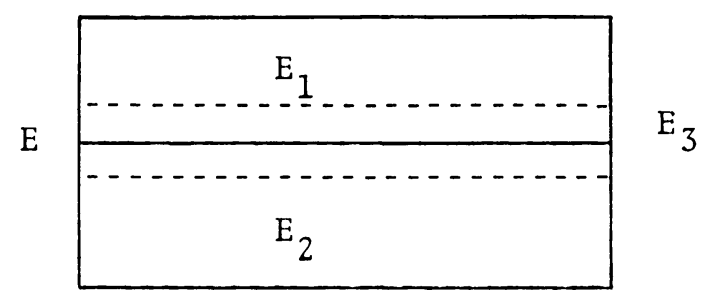

The result is immediate from the definition if we choose parallel transport on $E$ leaving $E_{i}, i=1,2$, invariant.

Proposition A4. Let $\xi: E^{n+k} \rightarrow M^{n}$ be the trivial bundle $M \times F \rightarrow M$. If $D^{k} \subset F$ is a small disc, then $T(\xi)=\left(i \Sigma^{k}\right)^{\chi(F)}$ and $T(\xi)_{*}: \pi_{r} C(M) \rightarrow \pi_{r} C(E)$ satisfies $T(\xi)_{*}=\chi(F) \cdot i_{r} \Sigma_{*}^{k}, i: M \times D^{k} \subset M \times F$.

Proof. (a) The result holds for $F=S^{k}$. This is obvious for $F=S^{1}$ by writing $M \times S^{1}=M \times D^{1} \cup M \times D^{1}$ and applying A3. The general result follows by induction by repeatedly applying A3 to $M \times S^{k}=M \times D^{k} \cup M \times D^{k}$.

(b) The result holds for $F=S^{p} \times D^{q}, p+q=k$. This follows from Al.

(c) The general result follows by taking a handle decomposition of $F$.

Proposition A5. Let $\xi: E \rightarrow M$ be a bundle with fibre $F$, and $\xi_{0}: E_{0} \rightarrow M$ the associated bundle with fibre $\partial F$. Then

$$
T \tau \cdot(\tau T)^{-1} \sim i \Sigma T_{0} \tau .
$$

Proof. (a) Define $\phi: C(M) \rightarrow C(M \times I)$, where $\phi(h)=\alpha(h) \cdot \beta(h)^{-1}$, $\alpha(h), \beta(h) \in \underline{A}^{c}\left(M \times I^{2}\right)$ :

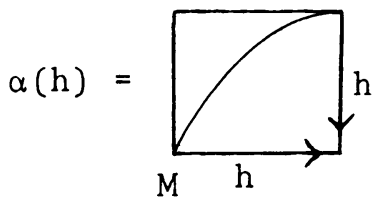

i.e. a $90^{\circ}$ rotation. (The arrow indicates the direction from 0 to 1 for $h$.) 

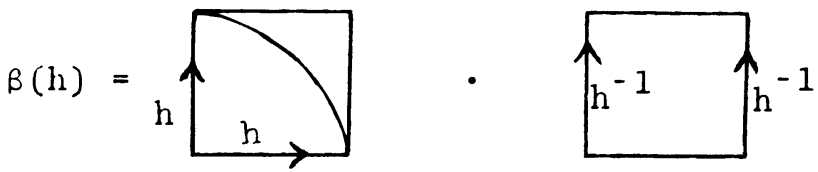

i.e. $90^{\circ}$ rotation in the first quadrant multiplied by id $\times h^{-1}$. Note that $\beta(h)$ can also be written.

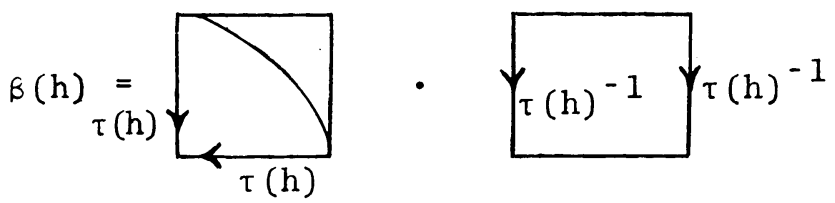

(b) Since $\gamma: C(M) \rightarrow \underline{A}_{s}\left(E \times I, E \times 0 \cup \partial_{-} E \times I\right)$ commutes with $\tau$, it is clear from the definition of $\sigma_{-}$that $T \tau \cdot(\tau T)^{-1} \sim i \phi T_{0} \tau, \phi: C\left(E_{0}\right) \rightarrow C\left(E_{0} \times I\right)$.

(c) Finally, it is not difficult to see that $\phi \sim \Sigma$. (The proof is given in the appendix.)

Corollary A6. Let $\xi: E \rightarrow M$ be a bundle with closed fibre $F$; then $T \tau \sim \tau T$.

Corollary A7. $\Sigma \tau=(\tau \Sigma)^{-1}$

Proof. For $E=M \times I, E_{0}=M \amalg M$ and $i \Sigma T_{0} \tau=(\Sigma \tau)^{2}$. Thus since $T=\Sigma$, we have from A5, $\Sigma \tau \cdot(\tau \Sigma)^{-1}=(\Sigma \tau)^{2}$.

COROllary A8. With the hypothesis of A5, if $T_{0}$ is trivial, $T \tau=\tau T$.

Definition. Let $\xi: E \rightarrow M$ be a bundle. Define $S(\xi): C(E) \rightarrow C\left(M \times D^{s}\right), s$ large, as follows: For $s$ sufficiently large, $\xi$ is homotopic to an embedding of $E$ in $M \times \check{D}^{s}$ with a normal (orthogonal or topological) disc bundle $\nu$. Let $E(\nu)$ be the total space of $\nu: E(\nu) \rightarrow E$. Let $S(\xi): C(E) \stackrel{T(\nu)}{\rightarrow} C(E(\nu)) \stackrel{i}{\rightarrow} C\left(M \times D^{s}\right)$. Then $S(\xi)$ is stably unique (up to homotopy). If $M=N \times I^{r}, S(\xi)$ clearly preserves the $r$ loop structure since $T(\nu)$ and $i$ do.

Proposition A9. Let $\xi: E \rightarrow M$ be the trivial bundle; then $S(\xi) \circ T(\xi): C(M) \rightarrow$ $C\left(M \times D^{k+s}\right)$ is $\left(\Sigma^{k+s}\right)^{x(F)}$.

Proof. The normal bundle $\nu$ of $E$ in $M \times D^{s+k}$ satisfies $\nu \mid M \times D^{k} \subset M \times F$ $=E$ is $M \times D^{k+s}$. The result follows from A4 and the definition of $S(\xi)$.

Proposition A10 (CF. [BL]). Let $\xi: E \rightarrow M$ be any orthogonal or topological disc bundle; then $S(\xi) \circ T(\xi)=\Sigma^{k+s}$.

Proof. In fact, $\xi \oplus \nu$ is the trivial disc bundle. Hence $E(\nu)=M \times D^{k+s} \subset M$ $\times D^{k+s}$ by the standard inclusion. Thus $i \circ T(\nu) \circ T(\xi)=i \circ T(\nu \oplus \xi)=\Sigma^{k+s}$.

Now let $\xi_{i}: E_{i}^{n+k} \rightarrow M^{n}, i=1,2$, be two bundles and $\phi: E_{1} \rightarrow E_{2}$ a fibre homotopy equivalence, and $\psi: E_{2} \rightarrow E_{1}$ a homotopy inverse. Then for $s$ sufficiently 
large, $i \psi: E_{2} \rightarrow E_{1} \times \dot{D}^{s}$ is homotopic to an embedding with normal (orthogonal or topological) disc bundle $\nu$. Again if $s$ is sufficiently large $i \phi: E_{1} \rightarrow \check{E}(\nu)$ is homotopic to an embedding with normal (orthogonal or topological) disc bundle. Since $E_{1} \subset E(\nu) \subset E_{1} \times \dot{D}^{s}$ is homotopic to the inclusion, for $s$ sufficiently large, the normal bundle of $E_{1}$ in $E(\nu)$ is trivial.

Let $F_{i}$ be the fibre of $E_{i}, i=1,2$. Then if $U \subset M$ is contractible, $\phi: U \times F_{1} \rightarrow$ $U \times F_{2} \subset U \times E\left(\nu \mid F_{2}\right)$ is a fibrewise map. Write $F_{2}^{\prime}=E\left(\nu \mid F_{2}\right)=$ fibre of $E(\nu) \rightarrow$ $E_{2} \rightarrow M$. Then $\phi \mid U$ defines a map $\phi_{\#}: U \rightarrow \operatorname{Maps}\left(F_{1}, F_{2}^{\prime}\right)$. For $s$ sufficiently large, $\pi_{i}\left(\operatorname{Maps}\left(F_{1}, F_{2}^{\prime}\right), E\left(F_{1}, F_{2}^{\prime}\right)\right)=0$ for $i \leqslant n$. Thus by induction over charts we may deform $i \phi$ fibrewise to a fibrewise embedding $\phi^{\prime}: E_{1} \subset E(\nu)$, as bundles over $M$.

In the Diff category it is immediate that $\phi^{\prime}$ has a fibrewise normal disc bundle in $E(\nu)$, which must be trivial by our choice of $\nu$ above. Thus $\phi^{\prime}$ extends to a fibrewise embedding of $E_{1} \times D^{s}$ in $E(\nu)$, as bundles over $M$.

In the Top category, we can assume that $F_{1}$ has a normal disc bundle in $F_{2}^{\prime}$ and hence, by the isotopy extension, $U \times F_{1}$ has a fibrewise normal disc bundle in $U \times F_{2}^{\prime}$ for each chart $U$ in $M$. Again this bundle is trivial, and we have a fibrewise embedding of $U \times F_{1} \times D^{s}$ in $U \times F_{2}^{\prime}$ extending $\phi^{\prime} \mid U$. Now on the overlap $U_{1} \cap U_{2}$ of two charts we have two fibrewise trivial normal disc bundles of $\left(U_{1} \cap U_{2}\right) \times F$ in $\left(U_{1} \cap U_{2}\right) \times F_{2}$. By shrinking the discs in one of these, we have an embedding $\left(U_{1} \cap U_{2}\right) \times F \times D^{s} \rightarrow\left(U_{1} \cap U_{2}\right) \times F \times D^{s}$ which defines a map of $\left(U_{1} \cap U_{2}\right) \times F \rightarrow E\left(D^{s}, D^{s}\right)$. Now from the fibration $C\left(S^{s-1}\right) \rightarrow \underline{A}\left(D^{s}\right) \rightarrow$ $E\left(D^{s}, D^{s}\right)$, and the fact that $\pi_{i} C\left(S^{s-1}\right)=0$ for $i \leqslant s-1$, we see that for $s$ sufficiently large $\left(U_{1} \cap U_{2}\right) \times F \rightarrow E\left(D^{s}, D^{s}\right)$ may be deformed to a map $\left(U_{1} \cap\right.$ $\left.U_{2}\right) \times F \rightarrow \underline{A}\left(D^{s}\right)$. Thus we may adjust the overlap of the bundles to give a fibrewise embedding of $\left(E_{1} \mid U_{1} \cup U_{2}\right) \times D^{s}$ in $E(\nu) \mid U_{1} \cup U_{2}$. By induction over the charts in $M$, we get a fibrewise embedding of $E_{1} \times D^{s}$ in $E(\nu)$ extending $\phi^{\prime}$. Thus we have proved:

Proposition A11. Let $\xi_{i}: E_{i}^{n+k} \rightarrow M^{n}, i=1,2$, be two bundles and $\phi$ a fibre homotopy equivalence. Then for $s$ sufficiently large, there is an $s$ disc bundle $E(\nu) \rightarrow E_{2}$ (orthogonal or topological, resp.) and a fibrewise embedding $\psi$ of $E_{1} \times D^{s}$ in $E(\nu)$, as bundles over $M$, such that $E_{1} \rightarrow E_{1} \times D^{s} \stackrel{\psi}{\rightarrow} E(\nu) \rightarrow E_{2}$ is fibrewise homotopic to $\phi$.

Referring to the notation above, if $\phi: E_{1} \rightarrow E_{2}$ gives a simple homotopy equivalence of $F_{1}$ to $F_{2}$, then $\overline{F_{2}^{\prime}-F_{1} \times D^{s}}$ is an $s$ cobordism and so is isomorphic to $F_{1} \times S^{s-1} \times I$. Thus $E(\nu)$ decomposes into two bundles over $M, E_{1} \times D^{s}$ and a bundle $E^{\prime}$ with fibre $F_{1} \times S^{s-1} \times I$, identified along $E_{1} \times S^{s-1}$. On the overlap of two coordinate charts the bundle $E^{\prime}$ is made by glueing $U_{1} \times F_{1} \times S^{s-1} \times I$ to $U_{2} \times F_{1} \times S^{s-1} \times I$ by a map $\alpha_{12}: U_{1} \cap U_{2} \rightarrow \underline{A}\left(F_{1} \times S^{s-1} \times I\right)$, where if $r_{0}$ : $\underline{A}\left(F_{1} \times S^{s-1} \times I\right) \rightarrow \underline{A}\left(F_{1} \times S^{s-1}\right)$ is the restriction to the " 0 end", $r_{0} \alpha_{12}$ is the glueing of $E^{\prime} \times S^{s-1}$ over $U_{1} \cap U_{2}$. If $\alpha_{12} \sim r_{0} \alpha_{12} \times \mathrm{id}_{1}$, then $E(\nu) \mid U_{1} \cup U_{2}$ is equivalent to $E_{1} \times D^{s} \mid U_{1} \cup U_{2}$ by a fibrewise isotopy of $\psi \mid U_{1} \cup U_{2}$. Note that

$$
\begin{aligned}
\alpha_{12} \circ\left(r_{0} \alpha_{12} \times \mathrm{id}_{I}\right)^{-1}: U_{1} \cap U_{2} & \rightarrow \underline{A}\left(F_{1} \times S^{s-1} \times I ; F_{1} \times S^{s-1} \times 0\right) \\
& \simeq C\left(F_{1} \times S^{s-1}\right) .
\end{aligned}
$$


So inductively assume that $E^{\prime}\left|\cup_{i<k} U_{i} \simeq E_{1} \times S^{s-1} \times I\right| \cup_{i<k} U_{i}$. Then if $\alpha_{k}$ : $\left(U_{k} \cap\left(\cup_{i<k} U_{i}\right)\right) \times F_{1} \times S^{s-1} \times I \rightarrow E^{\prime} \mid U_{k} \cap\left(\cup_{i<k} U_{i}\right)$ glues $E^{\prime} \mid U_{k}$ to $E^{\prime} \mid$ $\cup_{i<k} U_{i}$,

$$
\beta_{h}:\left(U_{k} \cap\left(\bigcup_{i<k} U_{i}\right)\right) \times F_{1} \times S^{s-1} \times I \rightarrow E_{1} \times S^{s-1} \times I \mid U_{k} \cap\left(\bigcup_{i<k} U_{i}\right)
$$

glues $E_{1} \times S^{s-1} \times I \mid U_{k}$ to $E_{1} \times S^{s-1} \times I\left|\cup_{i<k} U_{i}=E^{\prime}\right| \cup_{i<k} U_{i} ; \beta_{k}^{-1} \alpha_{k}$ is defined by a map

$$
U_{k} \cap\left(\bigcup_{i<k} U_{i}\right) \rightarrow \underline{A}\left(F_{1} \times S^{s-1} \times I ; F_{1} \times S^{s-1} \times 0\right) \simeq C\left(F_{1} \times S^{s-1}\right) .
$$

In the topological category if $F_{1}$ is $r$-connected, $\pi_{i} C\left(F_{1} \times S^{s-1}\right)=0$ for $i \leqslant r+$ $2, s \gg r$ [BLR]. Thus if $n \leqslant r+2, \psi$ will be fibrewise isotopic to a bundle equivalence over $M$. In the Diff category, we can only assert that if $F_{1}$ is $r$-connected then the inclusion of $D^{k+s-1}$ in $F_{1} \times S^{s-1}$ induces a homomorphism $\pi_{i} C\left(D^{k+s-1}\right) \rightarrow \pi_{i} C\left(F_{1} \times S^{s-1}\right)$ which is surjective for $i \leqslant 2 r-3$ and injective for $i \leqslant 2 r-4$ [BLR]. However, we can conclude that if $\xi_{i}, i=1,2$, are differentiable bundles, $\xi_{1}$ is trivial (which implies that $E^{\prime}$ has group $C\left(F_{1} \times S^{s-1}\right)$ ) and $F_{1}$ is $r$-connected; then $\psi$ is topologically isotopic to a topological bundle equivalence if $n \leqslant 2 r-3$. Thus we have proved:

Proposition A12. With the hypothesis of A11, and assuming the fibre of $\xi_{1}$ is $r$-connected, $r \geqslant n-2$, then in the topological category, $\psi$ may be taken to be a topological bundle equivalence.

Letting $C(\psi): C\left(E_{1} \times D^{s}\right) \rightarrow C(E(\nu))$ be the homomorphism induced by $\psi$ we have by Propositions Al and A4

COROLlaRY A13. (a) Under the hypothesis of $A 12, C(\psi) \circ \Sigma^{s} \circ T\left(\xi_{1}\right) \sim$ $T(\nu) \circ T\left(\xi_{2}\right)$ and $S\left(\xi_{1}\right) \circ T\left(\xi_{1}\right) \sim S\left(\xi_{2}\right) \circ T\left(\xi_{2}\right)$ in the topological category.

(b) If $\xi_{2}$ is trivial and $\chi\left(F_{1}\right)\left(=\chi\left(F_{2}\right)\right)$ is trivial, then $\Sigma^{s} \circ T\left(\xi_{1}\right) \sim 0$ and $S\left(\xi_{1}\right) \circ T\left(\xi_{1}\right) \sim 0$ in the topological category.

Proposition A14. Let $\xi: E^{n+k} \rightarrow M^{n}$ be a fibre homotopically trivial differentiable bundle with $r$-connected fibre $F, r \geqslant(n+3) / 2$. Then for $s$ sufficiently large, there is an orthogonal $D^{s}$ bundle $\nu$ over $E$ such that $E(\nu)$ is topologically equivalent to $M \times F \times D^{s}$ as a bundle over $M$.

COROLlaRY A15. Under the hypothesis of A14 in the topological category:

(a) $S(\xi) \circ T(\xi) \sim\left(\Sigma^{s}\right)^{x(F)}$.

(b) If $\chi(F)=0$, then $\Sigma^{s} \circ T(\xi) \sim 0$.

B. The spaces we deal with carry a natural (homotopy) involution whose symmetric, respectively antisymmetric, part provides different types of behavior. The success of our investigation comes from the possibility of regarding these parts differently. We collect below the elementary homotopy theory involved in what is actually the homotopy analogue of some trivial facts of linear algebra, and apply those considerations to the geometric situations we are concerned with. 
Properties of $Z\left(\frac{1}{2}\right)$ modules. Let $(M, \tau)$ be a commutative $Z\left(\frac{1}{2}\right)$ module with involution $\tau: M \rightarrow M$, i.e. $\tau^{2}=$ id and $\tau$ a $Z\left(\frac{1}{2}\right)$ linear map. $f:\left(M_{1}, \tau_{1}\right) \rightarrow\left(M_{2}, \tau_{2}\right)$ is called a morphism of $Z\left(\frac{1}{2}\right)$ modules with involution if $f$ is $Z\left(\frac{1}{2}\right)$ linear and $\tau_{2} \cdot f=f \cdot \tau_{1}$. Clearly we have:

(a) $M=M^{s} \oplus M^{a}, M^{s}=\{m \in M \mid \tau(m)=m\}, M^{a}=\{m \in M \mid \tau(m)=-m\}$.

(b) $f\left(M_{1}^{s}\right) \subset M_{2}^{s}, f\left(M_{1}^{a}\right) \subset M_{2}^{a}$ and $f=f^{s} \oplus f^{a}$ with $f^{s}=f / M_{1}^{s}$ and $f^{a}=f / M_{1}^{a}$.

(c) If $\cdots \rightarrow\left(M_{i}, \tau_{i}\right) \rightarrow^{f_{i}}\left(M_{i+1}, \tau_{i+1}\right) \rightarrow^{f_{i+1}}\left(M_{i+2}, \tau_{i+2}\right) \cdots$ is an exact sequence of modules with involution then

$$
\cdots M_{i}^{s} \stackrel{f_{i}^{s}}{\rightarrow} M_{i+1}^{s} \stackrel{f_{i+1}^{s}}{\rightarrow} M_{i+2}^{s} \stackrel{f_{i+2}^{s}}{\rightarrow} \ldots \quad \text { and } \quad \cdots M_{i}^{a} \stackrel{f_{i}^{a}}{\rightarrow} M_{i+2}^{a} \stackrel{f_{i+1}^{a}}{\rightarrow} M_{i+2}^{a} \rightarrow \cdots
$$

are exact sequences.

A weak commutative group with involution $(X, \tau)$ is an $H$ space $X$, whose multiplication satisfies up to homotopy the axioms of "commutative groups" and $\tau$ is an $H$ map with $\tau^{2}$ homotopic to identity. $f:\left(X_{1}, \tau_{1}\right) \rightarrow\left(X_{2}, \tau_{2}\right)$ is a morphism of weak commutative groups with involutions if $f$ is an $H$ map with $\tau_{2} \cdot f$ homotopic to $f \cdot \tau_{1}$. For any weak commutative group we have two canonical involutions $\mathrm{id}_{\boldsymbol{X}}$ and $\nu: X \rightarrow X$ with $\nu$ representing the "inverse" with respect to the multiplication on $X$.

Properties of $Z\left(\frac{1}{2}\right)$ commutative groups with involutions. A based pointed space (semisimplicial Kan complex) $(X, *)$ is called an odd space or a $Z\left(\frac{1}{2}\right)$ space if $\pi_{1}(X, *)$ is abelian and all homotopy groups $\pi_{i}(X, *)$ are $Z\left(\frac{1}{2}\right)$ modules. A weak commutative group with involution $(X, \tau)$ is a $Z\left(\frac{1}{2}\right)$ weak commutative group with involution if the space $(X, e)$ is a $Z\left(\frac{1}{2}\right)$ space. By localization to odd primes one can pass functorially from weak commutative groups with involution to $Z\left(\frac{1}{2}\right)$ weak commutative groups with involution.

Using "general homotopy theory" one can prove that:

(a) For any $(X, \tau)$, a $Z\left(\frac{1}{2}\right)$ weak commutative group with involution, there exist two $Z\left(\frac{1}{2}\right)$ weak commutative groups $X^{s}$ and $X^{a}$ together with a homotopy equivalence of weak commutative groups with involution $h(X, \tau):(X, \tau) \rightarrow\left(X^{s} \times X^{a}\right.$, $\left.\operatorname{id}_{X^{s}} \times \nu_{X^{a}}\right)$.

(b) If $f:\left(X_{1}, \tau_{1}\right) \rightarrow\left(X_{2}, \tau_{2}\right)$ is a morphism of $Z\left(\frac{1}{2}\right)$ weak commutative groups with involution, then there exist the morphisms $f^{s}:\left(X_{1}^{s}, \mathrm{id}_{X_{1}}\right) \rightarrow\left(X_{2}^{s}, \mathrm{id}_{X_{2}^{s}}\right)$ and $f^{a}$ : $\left(X_{1}^{a}, \nu_{X_{1}^{a}}\right) \rightarrow\left(X_{2}^{a}, \nu_{X_{2}^{a}}\right)$ of $Z\left(\frac{1}{2}\right)$ weak commutative groups with involution so that the following diagram is (homotopy) commutative:

$$
\begin{array}{ccc}
X_{1} & \rightarrow & X_{2} \\
h\left(X_{1}, \tau_{1}\right) \downarrow & & \\
X_{1}^{s} \times X_{1}^{a} & \rightarrow & X_{2}^{s} \times X_{2}^{a}
\end{array}
$$

(c) Let $\left(X_{1}, \tau_{1}\right) \stackrel{t}{\rightarrow}\left(X_{2}, \tau_{2}\right) \stackrel{g}{\rightarrow}\left(X_{3}, \tau_{3}\right)$ be a fibration with $\left(X_{i}, \tau_{i}\right) Z\left(\frac{1}{2}\right)$ weak commutative groups with involution and $f, g$ morphisms. Then $X_{1}^{s} \stackrel{f^{s}}{\rightarrow} X_{2}^{s} \stackrel{s^{s}}{\rightarrow} X_{3}^{s}$ and $X_{1}^{a} \stackrel{f^{a}}{\rightarrow} X_{2}^{a} \stackrel{g^{a}}{\rightarrow} X_{3}^{a}$ are fibrations. The geometric splitting we just described corresponds to the algebraic splitting previously described in the following sense; if $K$ is a space (semisimplicial Kan complex) then the set $[K, X]$ of homotopy classes of maps has a natural group structure of $Z\left(\frac{1}{2}\right)$ module with involution and $[K, X]^{s}=$ $\left[K, X^{s}\right]$, resp. $[K, X]^{a}=\left[K, X^{a}\right]$. 
(In the examples below we use the notation $\tilde{A} / A(M)=\tilde{A}(M) / A(M), \tilde{C} / C(M)$ $=\tilde{C}(M) / C(M)$.)

EXAMPLeS. (1) The involutions $\tau, \tilde{\tau}, \bar{\tau}$ on $C\left(M \times I^{r}\right), \tilde{C}\left(M \times I^{r}\right)$ and $\tilde{C} / C\left(M \times I^{r}\right)$. These will all be denoted abusively by $\tau$.

(2) On $A(M \times I)$ define the involution $\sigma$ by $\sigma(h)=(1 \times \lambda) \circ h \circ(1 \times \lambda), \lambda(t)=$ $1-t$. On $A\left(M \times I^{r}\right)$ we can define $r$ involutions, one for each $I$ factor in $M \times I^{r}$. However, they are all homotopic by involutions and will all be denoted by $\sigma$. Likewise on $C\left(M \times I^{r}\right)$ we may define involutions $\sigma$ with respect to the $I$ factors in $M \times I^{r}$. Again these are all homotopic by involutions to each other, but not to $\tau$. We also denote the corresponding involutions on $\tilde{A}\left(M \times I^{r}\right), \tilde{C}\left(M \times I^{r}\right)$, $\tilde{A} / A\left(M \times I^{r}\right), \tilde{C} / C\left(M \times I^{r}\right)$ by $\sigma$.

(3) On the loop space $\Omega X$ of any space $X$ we define the involution $\mu$ by $\mu(\alpha)(t)=\alpha(1-t), \alpha:[0,1] \rightarrow X$.

We observe that localized away from two, the following are weak commutative groups with involution:

(1) $C\left(M \times I^{r}\right), \tilde{C}\left(M \times I^{r}\right)$ for $r>0$, and $\tilde{C} / C\left(M \times I^{r}\right)$ for $r>1$, under $\tau$.

(2) $A\left(M \times I^{r}\right), \tilde{A}\left(M \times I^{r}\right), C\left(M \times I^{r}\right), \tilde{C}\left(M \times I^{r}\right)$ for $r>0$, and $\tilde{A} / A\left(M \times I^{r}\right), \tilde{C} / C\left(M \times I^{r}\right)$ for $r>1$, under $\sigma$.

(3) $\Omega X$ for $X$ an $H$ space, under $\mu$.

(4) $\Omega\left(\tilde{C} / C\left(M \times I^{r}\right)\right)$ for $r>0$, under $\sigma$ and $\tau . \Omega\left(\tilde{A} / A\left(M \times I^{r}\right)\right)$ for $r>0$, under $\sigma$.

We will denote the symmetric and antysymmetric parts under $\sigma$ by ()$^{s},()^{a}$; and under $\tau$ by ()$^{+},()^{-}$. Since $\sigma$ and $\tau$ commute on $C\left(M \times I^{r}\right)$, localized away from two, $C\left(M \times I^{r}\right)$ splits into the product of the four weak commutative groups: $C\left(M \times I^{r}\right)^{s+}, C\left(M \times I^{r}\right)^{s-}, C\left(M \times I^{r}\right)^{a+}, C\left(M \times I^{r}\right)^{a-}$.

Although $C\left(M^{n}\right)$ is not a weak commutative group, we will show it has the $[n / 2]-3$ type of a weak commutative group. We need

Proposition B1 [BLR]. If $n \geqslant 5, M^{n}$ is $k$-connected, and $M^{n}$ is obtained from $M_{0}^{n} \subset M^{n}$ by adding handles of index greater than $r, r \leqslant n-3$, then (1) $i$ : $C\left(M_{0}\right) \rightarrow C(M)$ is $\inf (2 r-3, r+k-2)$ - (resp. $\left.\inf (2 r-3, r+k-2, r+2)-\right)$ connected in the Diff (resp. Top) category; (2) $i: \tilde{A} / A\left(M_{0}\right) \rightarrow \tilde{A} / A(M)$ is $\inf (2 r-2, r+k-1)-(r e s p . \inf (2 r-2, r+k-1, r+3)-)$ connected in the Diff (resp. Top) category.

LEMMA B2. Let $M_{[n / 2]}$ be the union of all handles of $M^{n}$ of index $\leqslant[n / 2]$. Let $N^{n-1}=\partial M_{[n / 2]}$. Then $M$ is obtained from a collar neighborhood $N \times I \subset M$ by adding handles of index greater than $[n / 2]$.

Corollary B3. i: $C(N \times I) \rightarrow C(M)$ is $([n / 2]-2)$-connected and $i$ : $\tilde{A} / A(N \times I) \rightarrow \tilde{A} / A(M)$ is $([n / 2]-1)$-connected. (This result improves somewhat with the connectivity of $M$.)

This gives

Proposition B4. The following are fibrations of weak (commutative) groups with involution (when restricted to the Postnikov term indicated and $\operatorname{dim} M=n$ ).

(1)(a) $(\Omega A(M), \mu) \stackrel{\text { 促 }}{\rightarrow}(A(M \times I), \sigma) \stackrel{i}{\rightarrow}(C(M), \tau)($ up to $[n / 2]-3)$, 
(b) $(\Omega \tilde{A}(M), \mu) \rightarrow(\tilde{A}(M \times I), \sigma) \rightarrow(\tilde{C}(M), \tau)$,

(c) $(\Omega \tilde{A} / A(M), \mu) \rightarrow(\tilde{A} / A(M \times I), \sigma) \rightarrow(\tilde{C} / C(M), \tau)($ up to $[n-1]-2)$;

(2)(a) $\left(A\left(M \times I^{2}\right), \sigma\right) \stackrel{i}{\rightarrow}(C(M \times I), \sigma) \stackrel{r}{\rightarrow}(A(M \times I), \sigma)$,

(b) $\left(\tilde{A}\left(M \times I^{2}\right), \sigma\right) \rightarrow(\tilde{C}(M \times I), \sigma) \rightarrow(\tilde{A}(M \times I), \sigma)$,

(c) $\left(\tilde{A} / A\left(M \times I^{2}\right), \sigma\right) \rightarrow(\tilde{C} / C(M \times I), \sigma) \rightarrow(\tilde{A} / A(M \times I), \sigma)$ (up to $[n / 2]$ $-2)$.

Now using the fact that $(\Omega X)^{s}$ is trivial by definition, and the fact that $\tilde{C}(M)$ is contractible [BLR] we get from B4(1)(a)-(c):

Proposition B5. Localized away from two, the fibrations of B4(1) split:

(1)(a) $* \rightarrow A(M \times I)^{s} \rightarrow C(M)^{+}, \Omega A(M) \rightarrow A(M \times I)^{a} \rightarrow C(M)^{-}$,

(b) $* \rightarrow \tilde{A}(M \times I)^{s} \rightarrow *, \Omega \tilde{A}(M) \rightarrow \tilde{A}(M \times I)^{a} \rightarrow *$,

(c) ${ }^{*} \rightarrow \tilde{A} / A(M \times I)^{s} \rightarrow \tilde{C} / C(M)^{+}, \Omega \tilde{A} / A(M) \rightarrow \tilde{A} / A(M \times I)^{a} \rightarrow$ $\tilde{C} / C(M)^{-}$,

up to the Postnikov term indicated in B4.

Using the information from $\mathrm{B} 5$, we get

Proposition B6. Localized away from two, the fibrations of B4 (2) split:

(2)(a) $A\left(M \times I^{2}\right)^{s} \rightarrow C(M \times I)^{s+} \rightarrow *$,

$A\left(M \times I^{2}\right)^{a} \rightarrow C(M \times I)^{a-} \rightarrow A(M \times I)^{a}$,

$* \rightarrow C(M \times I)^{s-} \rightarrow A(M \times I)^{s}, * \rightarrow C(M \times I)^{a+} \rightarrow *$,

(b) $\tilde{A}\left(M \times I^{2}\right)^{s} \rightarrow * \rightarrow *, \tilde{A}\left(M \times I^{2}\right)^{a} \rightarrow * \rightarrow \tilde{A}(M \times I)^{a}$,

$* \rightarrow * \rightarrow \tilde{A}(M \times I)^{s}, * \rightarrow * \rightarrow *$,

(c) $\tilde{A} / A\left(M \times I^{2}\right)^{s} \rightarrow \tilde{C} / C(M \times I)^{s+} \rightarrow *$,

$\tilde{A} / A\left(M \times I^{2}\right)^{a} \rightarrow \tilde{C} / C(M \times I)^{a-} \rightarrow \tilde{A} / A(M \times I)^{a}$,

$* \rightarrow \tilde{C} / C(M \times I)^{s-} \rightarrow \tilde{A} / A(M \times I)^{s}, * \rightarrow \tilde{C} / C(M \times I)^{a+} \rightarrow *$

up to the Postnikov term indicated in B4.

Remark. If $M=N \times I^{r}$, then the fibrations of B4 and the splittings of B5, B6, deloop $r$ times, and the restrictions on the Postnikov term are only needed in the $r$ th delooping.

For the homotopy groups $\pi_{i}()=\pi_{i}() \otimes Z\left(\frac{1}{2}\right)$ we have (without restriction)

Proposition B5'. (1)(a) $\pi_{r}^{s} A(M \times I) \simeq{\pi_{r}^{+}}_{r}^{+}(M)$, and we have the exact sequence $\rightarrow \mathscr{F}_{i}^{a} A(M \times I) \rightarrow \mathbb{\pi}_{i}^{-} C(M) \rightarrow \mathscr{\pi}_{i}^{a} A(M) \rightarrow{\pi_{i-1}^{a}}_{i} A(M \times I) \rightarrow \cdots \rightarrow \pi_{0} A(M)$.

(b) $\pi_{r}^{s} \tilde{A}(M \times I) \equiv 0$ and $\pi_{i+1} \tilde{A}(M) \simeq \pi_{i}^{a} A(M \times I), i \geqslant 0$.

(c) $\pi_{r}^{s} \tilde{A} / A(M \times I) \simeq \pi_{r}^{+} \tilde{C} / C(M)$, and we have the exact sequence

$$
\begin{aligned}
\rightarrow \pi_{i}^{a} \tilde{A} / A(M & \times I) \rightarrow \pi_{i}^{-} \tilde{C} / C(M) \rightarrow \pi_{i} \tilde{A} / A(M) \\
& \rightarrow \pi_{i-1}^{a} \tilde{A} / A(M \times I) \rightarrow \cdots \rightarrow \pi_{1} \tilde{A} / A(M) \rightarrow 0 .
\end{aligned}
$$

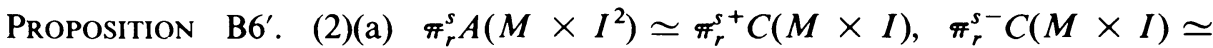
$\pi_{r}^{s} A(M \times I), \pi_{r}^{a+} C(M \times I) \equiv 0$, and we have the exact sequence

$$
\begin{aligned}
\rightarrow \pi_{i}^{a} A\left(M \times I^{2}\right) & \rightarrow \pi_{i}^{a-} C(M \times I) \rightarrow \pi_{i}^{a} A(M \times I) \\
& \rightarrow-\pi_{i-1}^{a} A\left(M \times I^{2}\right) \rightarrow \cdots \rightarrow \pi_{0}^{a} A(M \times I) .
\end{aligned}
$$



0 .

(b) $\pi_{r}^{s} \tilde{A}\left(M \times I^{2}\right)=\pi_{r}^{s} \tilde{A}(M \times I) \equiv 0$ and $\pi_{i+1}^{a} \tilde{A}(M \times I) \simeq \pi_{i}^{a} \tilde{A}\left(M \times I^{2}\right), i \geqslant$

(c) $\pi_{r}^{s} \tilde{A} / A\left(M \times I^{2}\right) \simeq \pi_{r}^{s+} \tilde{C} / C(M \times I), \pi_{r}^{s-} \tilde{C} / C(M \times I) \simeq \pi_{r}^{s} \tilde{A} / A(M \times I)$, ${\mathbb{F}_{r}}_{r}^{a+} \tilde{C} / C(M \times I) \equiv 0$, and we have the exact sequence

$$
\begin{aligned}
\rightarrow \mathbb{F}_{i}^{a} \tilde{A} / A(M & \left.\times I^{2}\right) \rightarrow \mathbb{\pi}_{i}^{a-} \tilde{C} / C(M \times I) \rightarrow \mathbb{F}_{i}^{a} \tilde{A} / A(M \times I) \\
& \rightarrow \mathbb{F}_{i-1}^{a} \tilde{A} / A\left(M \times I^{2}\right) \rightarrow \cdots \rightarrow \mathbb{F}_{1}^{a} \tilde{A} / A(M \times I) \rightarrow 0 .
\end{aligned}
$$

Let $B A(M \times I)$, resp. $B_{\#} A(M \times I)$, be the classifying spaces of $A(M \times I)$ under composition, resp. juxtaposition; and similarly for $\tilde{A}(M \times I), C(M \times I)$ and $\tilde{C}(M \times I)$.

Proposition B7. There is a homotopy equivalence

$$
\psi: B_{\#} A(M \times I) \rightarrow B A(M \times I)
$$

and similarly for $\tilde{A}(M \times I), C(M \times I)$ and $\tilde{C}(M \times I)$, which is natural with respect to the inclusions $j: A(M \times I) \rightarrow \tilde{A}(M \times I), j: \quad C(M \times I) \rightarrow \tilde{C}(M \times I), i$ : $A\left(M \times I^{2}\right) \rightarrow C(M \times I)$ and $i: \tilde{A}\left(M \times I^{2}\right) \rightarrow \tilde{C}(M \times I)$. If $M=N \times I^{r}$, then $\psi$ deloops $r$ times and commutes with $\sigma$ as defined by these I factors.

Corollary B8. The homotopy theoretic fibre $F_{C}$ of $B_{\# j} j: B_{\#} C(M \times I) \rightarrow$ $B_{\#} \tilde{C}(M \times I)$ is homotopy equivalent to $\tilde{C} / C(M \times I)$, and similarly for $F_{A}$ and $\tilde{A} / A(M \times I)$.

Since $\tau$ induces involutions on $B_{\#} C(M \times I)$ and $B_{\#} \tilde{C}(M \times I)$ with $B_{\#} j$ equivariant, it induces an involution on $F_{C}$.and hence a homotopy involution $\bar{\tau}$ on $\tilde{C} / C(M \times I)$.

Corollary B9. $i: \tilde{A} / A\left(M \times I^{2}\right) \rightarrow \tilde{C} / C(M \times I)$ satisfies io $\sim \bar{\tau} i$.

Proof. By naturality $\tilde{A} / A\left(M \times I^{2}\right) \sim F_{A} \rightarrow F_{i} F_{C} \sim \tilde{C} / C(M \times I)$ is homotopic to $i$. Since $B_{\#} j: B_{\#} A\left(M \times I^{2}\right) \rightarrow B_{\#} \tilde{A}\left(M \times I^{2}\right)$ commutes with $\sigma$ defined by the free $I$ factor, we get an action of $\sigma$ on $F_{A}$ such that $\tilde{A} / A\left(M \times I^{2}\right) \sim F_{A}$ is equivariant. Since $i \sigma=\tau i$ on $A\left(M \times I^{2}\right),(F i) \sigma=\tau(F i)$ on $F_{A}$. The result follows from the definition of $\bar{\tau}$.

Proof of Proposition B7. Let $R_{-s}=(-s, \infty)$, and for a base pointed space $(X, *)$ let $P X$ denote the space of paths which start from *. Define $T_{t}: M \times R \rightarrow$ $M \times R$ by $T_{t}(x, r)=(x, r+t)$. Define

$\phi: \operatorname{Emb}(M \times I, M \times[0, \infty) ; M \times 0 \cup \partial M \times I) \rightarrow P \operatorname{Emb}\left(M, M \times R_{-1}\right)$ by $\phi(h)_{t}(x)=T_{-t} h(x, t), \quad 0 \leqslant t \leqslant 1$; here we consider $M \times[0, \infty) \subset M \times$ $(-1, \infty)=M \times R_{-1} \subset M \times R$. Then we have a map of fibrations:

$$
\begin{array}{ccc}
A(M \times I) & \stackrel{\phi}{\rightarrow} & \Omega \operatorname{Emb}\left(M, M \times R_{-1}\right) \\
\downarrow & & \downarrow \\
E(M \times I, M \times[0, \infty) ; M \times 0 \cup \partial M \times I) & \stackrel{\phi}{\rightarrow} & P \operatorname{Emb}\left(M, M \times R_{-1}\right) \\
\downarrow & & \downarrow \\
E(M \times(1), M \times(0, \infty)) & \stackrel{\phi_{1}}{\rightarrow} & \operatorname{Emb}\left(M, M \times R_{-1}\right)
\end{array}
$$


where $\phi_{1}(h)(x)=T_{-1} h(x, 1)$. Now $\phi: A(M \times I) \rightarrow \Omega \operatorname{Emb}\left(M, M \times R_{-1}\right)$ sends juxtaposition to loop space addition, and $\phi_{1}$ is an equivalence. Since the total spaces are contractible,

$$
\begin{aligned}
\psi: B_{\#} A(M \times I) & \stackrel{B_{\#} \phi}{\rightarrow} \operatorname{Emb}\left(M, M \times R_{-}\right) \stackrel{\phi_{1}^{-1}}{\rightarrow} \operatorname{Emb}(M \times(1), M \times(0, \infty)) \\
& \simeq B A(M \times I)
\end{aligned}
$$

is a homotopy equivalence which satisfies all the properties.

ADDENDUM. There is a homotopy equivalence $\bar{\phi}: C(M \times I) \rightarrow \Omega C(M \times R)$ which is a homomorphism with respect to composition, and which commutes with $\tau$ (the composition and involution on $\Omega C(M \times R)$ being that induced from $C(M \times R))$. Similarly for $\tilde{C}(M \times I), \tilde{A}(M \times I)$ and $A(M \times I)$.

Proof. The restriction $r: C(M \times R) \rightarrow C \operatorname{Emb}(M, M \times R)$ is a homotopy equivalence, since the fibre $C(M \times R ; M \times 0)$ is contractible. Define

$$
\bar{\phi}: C(M \times I) \rightarrow \Omega C\left(M \times R_{-1}\right) \text { by } \bar{\phi}(h)=T_{-t} h T_{t} .
$$

Then $\Omega(r) \bar{\phi}=\phi$; and since $\phi$ is a homotopy equivalence (see proof above), $\bar{\phi}$ is a homotopy equivalence. (Note we take $h(x, r)=(x, r)$ for $r<0$ or $r>1$.)

C. Consequences of stability. In this section we assume $\Sigma: C\left(M^{n}\right) \rightarrow C\left(M^{n} \times I\right)$ is an $\omega(n)$ homotopy equivalence where $\omega(n+1)>\omega(n)$ and $\lim _{n \rightarrow \infty} \omega(n)=\infty$. According to the result of Hatcher $[\mathbf{H}]$ as corrected by Hsiang we can take $\omega(n) \sim n / 6$ in the PL category. This implies we can take $\omega(n) \sim n / 6$ in the Top category and $\omega(n) \sim n / 12$ in the Diff category [BL].

Since $\Sigma$ anticommutes with $\tau$ and $\Sigma: C(M \times I) \rightarrow C\left(M \times I^{2}\right)$ clearly commutes with $\sigma$, we have

C1. $\Sigma: C(M \times I)^{a-} \rightarrow C\left(M \times I^{2}\right)^{a+}$ and hence by B6(a) and stability, $C(M \times I)^{a-}$ is $\omega(n+1)$-connected, and thus by B6(a), (c), B6'(a), (c):

C2. (a) $\Omega A(M \times I)^{a} \rightarrow A\left(M \times I^{2}\right)^{a}$ is $\omega(n+1)$-connected.

(b) $\Omega \tilde{A} / A(M \times I)^{a} \rightarrow \tilde{A} / A\left(M \times I^{2}\right)^{a}$ is $\inf (\omega(n+1)+1,[n / 2]-2)$ connected.

(c) $\pi_{i+1}^{a} A(M \times I) \approx \mathbb{F}_{i}^{a} A\left(M \times I^{2}\right), 0 \leqslant i \leqslant \omega(n+1)$.

(d) $\pi_{i+1}^{a} \tilde{A} / A(M \times I) \approx \pi_{i}^{a} \tilde{A} / A\left(M \times I^{2}\right), 0<i<\omega(n+1)+1$ and hence by induction (since $\left.\pi_{0} \tilde{A} / A\left(M \times I^{r}\right)=0\right), \pi_{i}^{a} \tilde{A} / A(M \times I)=0, i \leqslant \omega(n+1)$.

Applying these results to $\mathrm{B}^{\prime}$ one gets

C3. $\pi_{i}^{-} \tilde{C} / C(M) \rightarrow \pi_{i} \tilde{A} / A(M)$ is an isomorphism for $i<\omega(n+1)$ and an epimorphism for $i=\omega(n+1)+1$.

Now note that stability implies that $C(M)$ is a weak commutative group up to $\omega(n)$, and hence $\pi_{*}^{ \pm} C(M) \simeq \pi_{*} C(M)^{ \pm}$up to $\sup (\omega(n),[n / 2]-3)$. Thus we have (writing $X_{\text {odd }}$ for $X$ localized away from two) from B5, B7 and C3:

THEOREM C4. For the $\omega(n)$ Postnikov terms we have the $\omega(n)$ equivalences

$$
C(M)^{-} \sim(\Omega \tilde{A} / A(M))_{\text {odd }}, \quad C(M)^{+} \sim A(M \times I)^{s} .
$$

These equivalences are natural with respect to inclusions $M^{\prime n} \subset M^{n}$, and if $M=N$ $\times I^{r}$ they deloop $r$ times. 
Since $\Sigma^{2}$ commutes with $\tau, \mathrm{C} 4$ implies

C5. (a) $A(M \times I)^{s}$ is $\omega(n)$ equivalent to $A\left(M \times I^{2 r+1}\right)^{s}$ and $\mathrm{C} 1$ and $\mathrm{B} 6$ imply

C5. (b) The following are $\omega(n+1)$ equivalences:

$$
\begin{gathered}
C(M \times I)^{-} \sim A(M \times I)^{s}, \quad C(M \times I)^{+} \sim A\left(M \times I^{2}\right)^{s}, \\
A(M \times I)^{a} \sim \tilde{A}(M \times I)^{a}, \quad \tilde{A}(M \times I)^{s} \sim^{*} .
\end{gathered}
$$

In particular, since $A(M \times I)_{\text {odd }} \simeq A(M \times I)^{s} \times A(M \times I)^{a}$, we have:

Proposition C6. There is an $\omega(n+1)$ equivalence $A(M \times I)_{\text {odd }} \sim \tilde{A}(M \times I)_{\text {odd }}$ $\times C(M \times I)^{-}$.

Note that Proposition $\mathrm{C} 6$ is equivalent to $\mathrm{C} 4$ (with $M \times I$ for $M$ ) together with a cross-section of the fibration $\Omega \tilde{A} / A(M \times I)_{\text {odd }} \rightarrow A(M \times I)_{\text {odd }} \rightarrow \tilde{A}(M \times I)_{\text {odd }}$ or equivalently that the quotient map $\tilde{A}(M \times I) \rightarrow \tilde{A} / A(M \times I)$ localized away from two is homotopically trivial. This is almost true for $\tilde{A}(M)$. To explain this let us denote by $\tilde{\mathcal{K}}(M)$, respectively $\tilde{\mathcal{H}}(M)$, the block versions of $\mathscr{\mathcal { H }}(M)$, respectively $\mathcal{H}(M)$; a typical $k$-simplex being a face preserving simple homotopy equivalence, respectively, which restricts to the identity on $\Delta[k] \times \partial M, h: \Delta[k] \times M \rightarrow \Delta[k] \times$ $M$. It is immediate that the inclusions $\mathcal{H}(M) \subset \tilde{\mathcal{H}}(M)$, respectively $\mathscr{F}(M)$ $\subset \tilde{\mathcal{F}}(M)$, are homotopy equivalences.

THEOREM C7. (a) The projection $q: \tilde{A}(M) \rightarrow \tilde{A} / A(M)$ when localized away from two and restricted to the $\omega(n)+1$ Postnikov term factors through $q^{\prime}: \tilde{\mathcal{H}}(M) \rightarrow$ $\tilde{A} / A(M)$ up to homotopy.

(b) Moreover, there is a map $\beta:[\tilde{\mathcal{F}}(M)]_{\omega(n)+1} \rightarrow[\tilde{C} / C(M)]_{\omega(n)+1}$ such that $q^{\prime}$ and $r \beta$ are homotopic when localized away from two, where $r: \tilde{C} / C(M) \rightarrow \tilde{A} / A(M)$ is the restriction map.

Corollary C8. Localized away from two and restricted to the $\omega(n)$ Postnikov term, $\Omega \tilde{\tilde{F}} / A(M) \simeq \Omega \tilde{\mathcal{H}} / \tilde{A}(M) \times C(M)^{-}$.

Since $\tilde{A}(M) \rightarrow \tilde{\mathcal{H}}(M)$ factors through $\tilde{\mathcal{H}}(M)$,

Corollary C9. Localized away from two and restricted to the $\omega(n)$ Postnikov term, $\Omega \tilde{\mathcal{H}} / A(M) \simeq \Omega \tilde{\mathcal{H}} / \tilde{A}(M) \times C(M)^{-}$.

In order to prove Theorem $\mathrm{C} 7$ we need the following results:

(i) Localized away from two $\cdots \times \operatorname{id}_{S^{2 n}}: \tilde{A} / A(M) \rightarrow \tilde{A} / A\left(M \times S^{2 n}\right)$ is an $\omega(n)+1$ equivalence.

Consider the fibre $\tilde{A}\left(M \times D^{r} ; M\right)$ of the restriction $r: \tilde{A}\left(M \times D^{r}\right)$ $\rightarrow \widetilde{\mathrm{Emb}}\left(M, M \times D^{r}\right)$, and let $\underline{A}^{0}\left(M \times D^{r}\right)$ be the subgroup of $\underline{A}\left(M \overline{\times} D^{r}: M\right)$ of orthogonal (resp. topological) bundle automorphisms of the trivial bundle $M \times$ $D^{r}$, then:

(ii) The inclusion $\underline{A}^{0}\left(M \times D^{r}\right) \rightarrow \tilde{A}\left(M \times D^{r} ; M\right)$ is a homotopy equivalence in the Diff category and $(r-1-n)$-connected in the Top category.

Let $\widetilde{\mathrm{Emb}^{0}}\left(M, M \times D^{r}\right)$ be the subspace of $\widetilde{\mathrm{Emb}}\left(M, M \times D^{r}\right)$ of embeddings which are simple homotopy equivalences. 
(iii) If $p$ : $\widetilde{\operatorname{Emb}}\left(M, M \times D^{r}\right) \rightarrow \tilde{\mathcal{H} C}(M)$ is the composition with projection of $M \times D^{r}$ on $\overline{M, \text { then }} p$ is $(r-1-\bar{n})$-connected.

(iv) The forgetful map $\theta: \tilde{C} / C\left(M \times D^{2 r}\right) \rightarrow \underline{\tilde{A}} / \underline{A}\left(M \times D^{2 r+1}\right)$ is $(2 r-n-2)$ connected.

Assume we have proved (i)-(iv).

Proof of (a). The image of $r: \tilde{A}\left(M \times D^{r}\right) \rightarrow \widetilde{\operatorname{Emb}}\left(M, M \times D^{r}\right)$ is a union of components of $\widetilde{E m b}^{0}\left(M, M \times D^{r}\right)$, and $r$ induces

$$
\bar{r}: \underline{\tilde{A}}\left(M \times D^{r}\right) / \underline{A}^{0}\left(M \times D^{r}\right) \rightarrow \widetilde{\mathrm{Emb}}^{0}\left(M, M \times D^{r}\right),
$$

which by (ii) is a homotopy equivalence ( $r-1-n$ equivalence) onto these components. Consider the commutative diagram, where $\sim$ will mean an equivalence of $\omega(n)+1$ Postnikov terms when localized away from two and for $r$ sufficiently large:

(I)

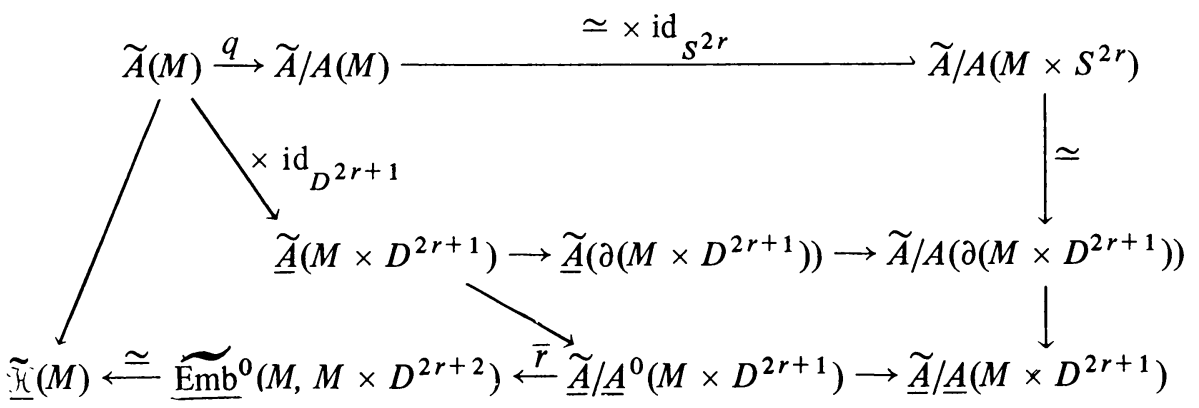

The equivalence follows from Theorems $\mathrm{C} 4, \mathrm{C} 5$ and B1. By commutativity of the diagram, (a) follows by reversing the equivalences and $\bar{r}$ (sending the extra components of $\mathrm{Emb}^{0}\left(M, M \times D^{2 r+1}\right)$ trivially).

Proof of (b). Let $\alpha: \tilde{\mathcal{H}}(M) \rightarrow \tilde{A} / \underline{A}\left(M \times D^{2 r+1}\right)$ be the map obtained by reversing $\bar{r}$ and the equivalence provided by (iii), in the bottom line of (I). Let $\beta=\left(\Sigma^{2 r}\right)^{-1} \theta^{-1} \alpha$. For $r$ sufficiently large this is defined. The result now follows from (I) and the commutative diagram

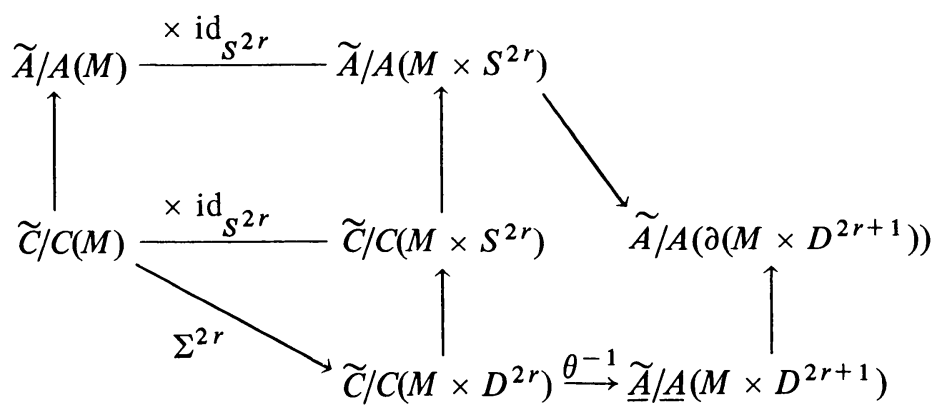


REMARK. Note that for any manifold $N$, the diagram

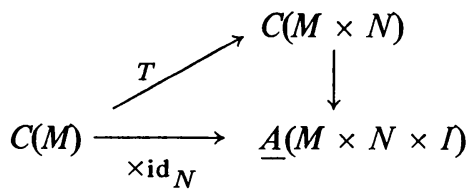

is homotopy commutative. Hence

(III)

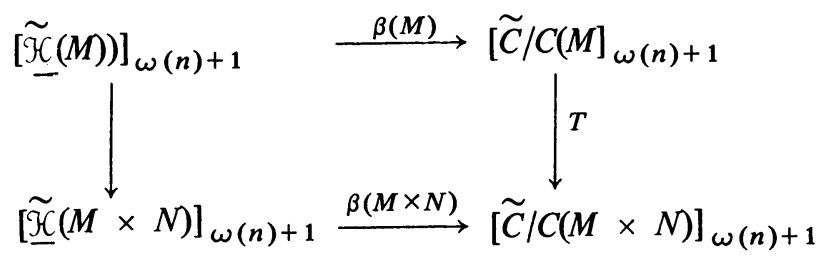

is homotopy commutative.

To have the proof of Theorem C7 finished, it remains to check (i)-(iv).

ProOF OF (i). This is immediate from $\mathrm{C} 3$ and the commutativity of the diagram

$$
\begin{array}{ccc}
\pi_{i}-\tilde{C} / C(M) & \stackrel{\times S^{2 k}}{\rightarrow} & \pi_{i}-\tilde{C} / C\left(M \times S^{2 k}\right) \\
\downarrow & & \downarrow \\
\pi_{i} \tilde{A} / A(M) & \stackrel{\times S^{2 k}}{\rightarrow} & \pi_{i} \tilde{A} / A\left(M \times S^{2 k}\right)
\end{array}
$$

Note that $\times S^{2 k}$ on $\tilde{C} / C(M)$ is the transfer for the trivial bundle $M \times S^{2 k} \rightarrow M$ and hence commutes with $\tau$.

Proof OF (ii). Consider the fibration

$$
\begin{aligned}
\underline{\tilde{A}}\left(M \times S^{r-1} \times I ; M\right. & \left.\times S^{r-1} \times 0\right) \rightarrow \underline{\tilde{A}}\left(M \times D^{r} ; M\right) \\
\rightarrow & \underline{\tilde{E}}\left(M \times D_{e}^{r}, M \times \dot{D}^{r} ; M\right)
\end{aligned}
$$

where, for $\partial M \neq 0, \tilde{A}\left(M \times D^{r} ; M\right)$ must be replaced by the homotopy equivalent subgroup of the block automorphisms $h$ with $h\left(\partial M \times D_{e}^{r}\right) \subset \partial M \times \dot{D}^{r}$. The result in the Diff category is immediate from the tubular neighborhood theorem and the fact that $\tilde{A}\left(M \times S^{r-1} \times I ; M \times S^{r-1} \times 0\right)$ is contractible.

In the Top category (cf. [L]) we have the fibration

$$
\underline{\tilde{E}}\left(M \times D_{\varepsilon}^{r}, M \times \dot{D}^{r} ; M\right) \rightarrow \underline{\tilde{I}}\left(M \times D_{e}^{r}, M \times \dot{D}^{r}\right) \rightarrow \tilde{\operatorname{Im}}\left(M, M \times \dot{D}^{r}\right) .
$$

Now $\tilde{I m}\left(M, M \times \dot{D}^{r}\right), r \geqslant 3$, is a space of sections of a fibre space over $M$ with fibre $\bar{G} / G_{r}$, and $\tilde{I}\left(M \times D_{e}^{r}, M \times \dot{D}^{r}\right)$ is a space of sections of a principal fibration with fibre Top. Thus $\underline{\mathbb{m}}\left(M, M \times D^{r}\right)$ is $(r-1-n)$-connected, and

$$
\underline{\tilde{m}}\left(M \times \overline{D_{\varepsilon}^{r}}, M \times D^{r}\right)=\operatorname{Maps}(M, \text { Top }) \text {. }
$$

Since $\underline{A}^{0}\left(M \times D^{r}\right)=\operatorname{Maps}(M$, Top $r)$ and $\underline{A}^{0}\left(M \times D^{r}\right) \rightarrow \widetilde{\operatorname{Im}}\left(M \times D^{r}, M \times \dot{D}^{r}\right)$ corresponds to the map $\operatorname{Maps}\left(M\right.$, Top $\left._{r}\right) \rightarrow \operatorname{Maps}(M$, Top) induced by the inclusion of Top $r$ in Top, the result follows from the fact that $\pi_{i}$ (Top, Top $\left.{ }_{r}\right)=0$ for $i<r$. 
Proof OF (iii). $\pi_{j} \tilde{E}\left(M, M \times D^{r}\right)=$ concordance classes of proper embeddings $f$ : $D^{j} \times M \rightarrow D^{j} \times \bar{M} \times D^{r}$ such that $f \mid \partial D^{j} \times M=$ inclusion. If $j<r-n-1$, then $j+n+r \geqslant 2(j+n)+1$, and the result follows from the Whitney embedding theorem.

ProOF OF (iv). From the map of fibrations:

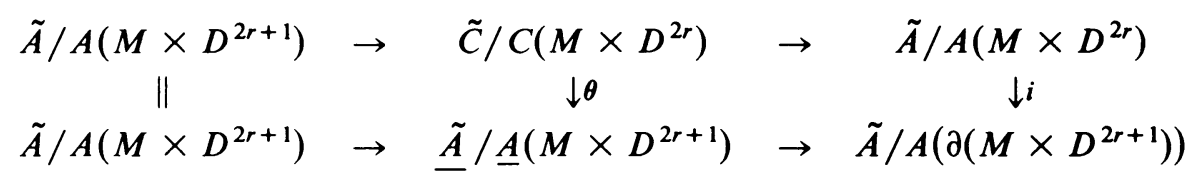

and Proposition B1, the connectivity of $\theta$ follows.

Using the stability assumption we can now interpret a number of the results of $\S \mathrm{A}$ in the stability range.

Theorem C10. On the $\omega(n)$ Postnikov term:

(a) If $\xi: E \rightarrow M^{n}$ is any orthogonal or topological disc bundle, $T(\xi): C(M) \sim$ $C(E)$.

(b) If $\xi$ : $E \rightarrow M$ is a trivial bundle and $\chi(F)=0, T(\xi)$ is trivial.

(c) If $\xi: E \rightarrow M$ is a trivial bundle and $\chi(F)=2$, then localized away from two, $T(\xi): C(M) \sim C(E)$.

(d) If $\xi: E \rightarrow M$ is a fibre homotopically trivial bundle and $F$ is $r$-connected $(r \geqslant n-2$ if $\xi$ is topological, $r \geqslant(n+3) / 2$ if $\xi$ is differentiable), then in the topological category:

(1) if $\chi(F)=0, T(\xi)$ is trivial;

(2) if $\chi(F)=2$, then localized away from two, $T(\xi): C(M) \sim C(E)$.

Proof. (a), (b), (c), (d) follow respectively from A10, A9, A9(b) above and A13 and A15.

Definition. We will say that a disc bundle is fibre homotopically trivial if its associated sphere bundle is fibre homotopically trivial. A disc bundle will be called stably fibre homotopically trivial if the bundle plus a trivial bundle is a fibre homotopically trivial disc bundle.

Corollary C11. Let $\xi: E \rightarrow M$ be a topological $k$ disc bundle which is stably fibre homotopically trivial. Then on the $\omega(n)$ Postnikov term:

$$
T(\xi) \tau \sim(\tau T(\xi))^{(-1)^{k}} .
$$

Proof. Take $s$ large and such that $k+s$ is even. Then $\xi \oplus 1_{s}: E \times D^{s} \rightarrow M$ is fibre homotopically trivial, i.e. its associated sphere bundle $\left(\xi \oplus 1_{s}\right)_{0}:\left(E \times D^{s}\right)_{0} \rightarrow$ $M$ is a fibre homotopically trivial $S^{k+s-1}$ bundle.

Since $k+s-1$ is odd, $\chi\left(S^{k+s-1}\right)=0$ and $T\left(\left(\xi \oplus 1_{s}\right)_{0}\right)$ is trivial by C10(d). Hence by A8, $T\left(\xi \oplus 1_{s}\right) \tau=\tau T\left(\xi \oplus 1_{s}\right)$. But $T\left(\xi \oplus 1_{s}\right) \sim \Sigma^{s} T(\xi)$ and $\Sigma^{s}$ is an isomorphism (on the $\omega(n)$ Postnikov term). Hence the result follows from A7.

We wish to prove a version of $\mathrm{Cl1}$ in the Diff category. We will only be able to do this localized away from two. We first need to develop a theorem from [BL].

Let $X$ be a finite complex and $N^{n}(X)$ a regular neighborhood of $X$ in some large Euclidean space $E^{n}$. Then $N^{n}(X)$ is a smooth compact manifold and $N^{n+1}(X)=$ $N^{n}(X) \times I$. Let $P(X)=\lim _{\rightarrow} C\left(N^{n}(X)\right)$, under $\Sigma, P_{T}(X)=\lim _{\rightarrow_{n}} C^{\text {Top }}\left(N^{n}(X)\right)$ and 
$P_{D}(X)=\lim _{\rightarrow} C^{\text {Diff }}\left(N^{n}(X)\right)$. Then from [BL] we have

TheOREM C12. $\pi_{i}\left(P_{T}(X) / P_{D}(X)\right)=H_{i}(X ; \mathscr{F})$, where $H_{i}(, \mathscr{F})$ is a homology theory with coefficients in the spectrum $\mathscr{F}$.

Now let $M^{n}$ be a smooth compact manifold and $E(\nu)$ its normal bundle in some large Euclidean space $E^{n+s}$. Then $E(\nu)=N^{n+s}(M)$. Then on the $\omega(n)$ Postnikov term we have $C(M) \stackrel{T(\nu)}{\rightarrow} C(E(\nu))=C\left(N^{n+s}(M)\right) \stackrel{\Sigma^{\infty}}{\rightarrow} P(M)$ is an isomorphism which is natural with respect to inclusions $M^{\prime n} \subset M^{n}$ and with respect to the forgetful map from the Diff category to the Top category. Hence if $M^{n}=M_{1}^{n} \cup$ $M_{2}^{n}$ with $M_{1} \cap M_{2}$ an $n-1$ manifold, we have

C13. For $i \leqslant \omega(n)$, there is a Mayer-Vietoris sequence

$$
\begin{array}{r}
\rightarrow \pi_{i} C^{\text {Top }} / C^{\text {Diff }}\left(\left(M_{1} \cap M_{2}\right) \times I\right) \rightarrow \pi_{i} C^{\text {Top }} / C^{\text {Diff }}\left(M_{1}\right) \oplus \pi_{i} C^{\text {Top }} / C^{\text {Diff }}\left(M_{2}\right) \\
\rightarrow \pi_{i} C^{\text {Top }} / C^{\text {Diff }}(M) \rightarrow \pi_{i-1} C^{\text {Top }} / C^{\text {Diff }}\left(\left(M_{1} \cap M_{2}\right) \times I\right) \rightarrow \ldots
\end{array}
$$

THEOREM C14. Let $\xi: E \rightarrow M^{n}$ be an orthogonal $S^{2 k}$ bundle over the smooth manifold $M, 2 k-1 \geqslant(n+3) / 2$, which is fibre homotopically trivial. Then on the $\omega(n)$ Postnikov term, $T: C^{\text {Diff }}(M) \rightarrow C^{\text {Diff }}(E)$ is an equivalence when localized away from two.

Proof. Take a handle decomposition of $M$. Then over each handle $D^{i} \times D^{n-i}, \xi$ is trivial. Hence $T\left(\xi\left(D^{i} \times D^{n-i}\right)\right)$ is an equivalence in both the Diff and Top categories and hence on $C^{\text {Top }} / C^{\text {Diff }}\left(D^{i} \times D^{n-i}\right)$. The same is true for $S^{i-1} \times$ $D^{n-i} \times I, S^{i-1} \times D^{n-i}$ the boundary of the handle. Hence by induction over the handles and C13, T: $C^{\text {Top }} / C^{\text {Diff }}(M) \rightarrow C^{\text {Top }} / C^{\text {Diff }}(E)$ is an equivalence (on the $\omega(n)$ Postnikov term when localized away from two). By ClO(d), T: $C^{\text {Top }}(M) \rightarrow$ $C^{\text {Top }}(E)$ is an equivalence. Hence by the 5-lemma, $T: C^{\text {Diff }}(M) \rightarrow C^{\text {Diff }}(E)$ is an equivalence.

COROllary C15. Let $\xi: E \rightarrow M$ be an orthogonal $k$ disc bundle over a smooth $n$-manifold which is stably fibre homotopically trivial. Then localized away from two and on the $\omega(n)$ Postnikov term there is an equivalence $\theta(\xi): C(M) \rightarrow C(E)$ such that $\theta(\xi) \tau \sim(\tau \theta(\xi))^{(-1)^{k}}$.

Proof. Take $s$ large and such that $k+s$ is odd. Then $\xi \oplus 1_{s}: E\left(\xi \oplus 1_{s}\right) \rightarrow M$ is fibre homotopically trivial, i.e. its associated sphere bundle $\left(\xi \oplus 1_{s}\right)_{0}: E\left(\xi \oplus 1_{s}\right)_{0} \rightarrow$ $M$ is a fibre homotopically trivial $S^{k+s-1}$ bundle. Since $k+s-1$ is even, $T$ : $C^{\text {Diff }}(M) \rightarrow C^{\text {Diff }}\left(E\left(\xi \oplus 1_{s}\right)_{0}\right)$ is an equivalence by C14. Let $i: E\left(\xi \oplus 1_{s-1}\right) \subset$ $E\left(\xi \oplus 1_{s}\right)_{0}$ be the natural smooth embedding. Then $i$ induces an equivalence on $C^{\text {Diff }}$ by B1. Define

$$
\theta(\xi): C(M) \stackrel{T\left(\xi \oplus 1_{s}\right)_{0}}{\rightarrow} C\left(E\left(\xi \oplus 1_{s}\right)_{0}\right) \stackrel{i^{-1} / 2}{\rightarrow} C\left(E\left(\xi \oplus 1_{s-1}\right)\right) \stackrel{\left(\Sigma^{-1}\right)^{-1}}{\rightarrow} C(E(\xi)) .
$$

This is well-defined equivalence in the $\omega(n)$ Postnikov term and when localized away from two, provided $s$ is sufficiently large. Since the first two maps commute with $\tau$, while $\Sigma^{s-1} \tau=\left(\tau \Sigma^{s-1}\right)^{(-1)^{s-1}}$, and $s-1$ has the same parity as $k$, the result follows. 
REMARKS. (a) If $\xi$ is trivial, then $\theta(\xi)=T(\xi)$.

(b) In the Top category, $\theta(\xi)=T(\xi)$.

In a certain sense all sphere bundles with the same first Stiefel-Whitney class are stably fibre homotopically isomorphic when localized at zero. To make this precise let $B G$ be the classifying space for stable spherical fibrations and for any such fibrations $\xi$ over $X$, let $|\xi|: X \rightarrow B G$ be classifying maps and $\xi^{\#}: \pi_{2}(X) \rightarrow \pi_{2}(B G)$ $=Z_{2}$ be the homomorphism induced for the second homotopy group.

LEMMA C16. If $X$ is a finite $C W$ complex and $\xi_{1}, \xi_{2}$ are two stable spherical fibrations over $X$ with the same first Stiefel-Whitney class, then:

(a) If $\xi_{1}^{\#} \neq \xi_{2}^{\#}$ for any $k \geqslant 1$ there exists $\phi: Y \rightarrow X$ with $Y$ a finite $C W$ complex so that $\phi_{*}: \pi_{1}(Y) \rightarrow \pi_{1}(X)$ is an isomorphism, $\pi_{i}(\phi) \otimes Q=0$ for $2 \leqslant i \leqslant k$ and $\phi^{*} \xi_{1}$ and $\phi^{*} \xi_{2}$, the pull backs of $\xi_{1}$ and $\xi_{2}$ by $\phi$, are stably fibre homotopically isomorphic.

(b) If $\xi_{1}^{\#}=\xi_{2}^{\#}$, there exist $\hat{\xi}_{1}, \hat{\xi}_{2}$ over $X \times S^{2}$ whose restrictions to $X \times\{p\}$ are $\xi_{1}$, respectively $\xi_{2}$, and $\hat{\xi}_{1}^{\#} \neq \hat{\xi}_{2}^{\#}$.

Proof. (a) Let $B S G$ be the classifying space for oriented stable spherical fibrations and $i: B S G \rightarrow B G$ the map defined by considering any oriented stable spherical fibration as a stable spherical fibration.

Clearly there exists $|\eta|: X \rightarrow B S G$ so that $\left|\xi_{1}\right|+i \circ|\eta| \sim\left|\xi_{2}\right|$ and $\eta^{\#}: \pi_{2}(X) \rightarrow$ $\pi_{2}(B G)=Z_{2}$ nontrivial, hence surjective. Let $L$ be the homotopy theoretic fibre of $|\eta|$. It is not hard to show that $L$ has the homotopy type of a CW complex with finitely many cells in each dimension. Let $Y=L^{(k+1)}$ be the $(k+1)$ skeleton.

(b) Let $|u|: S^{2} \rightarrow B G$ be the generator of $\pi_{2}(B G)=Z_{2}$. Consider $\left|\hat{\xi}_{1}\right|$, respectively $\left|\hat{\xi}_{2}\right|$, the compositions $X \times S^{2} \stackrel{\left|\xi_{1}\right| \times|u|}{\rightarrow} B G \times B G \stackrel{\mu}{\rightarrow} B G$, respectively $X \times S^{2} \stackrel{\left|\xi_{2}\right| \times 0}{\rightarrow} B G \times B G \stackrel{\mu}{\rightarrow} B G$, where $\mu$ denotes the loop space multiplication in $B H$ and 0 the constant map.

THEOREM C17. Let $\xi: E \rightarrow M^{n}$ be a topological (respectively orthogonal) oriented $k$ disc bundle. Then on the $\omega(n)$ Postnikov term and localized at zero, $T(\xi) \tau \simeq$ $(\tau T(\xi))^{(-1) k}\left(\operatorname{resp} . \theta(\xi) \tau \sim(\tau \theta(\xi))^{(-1)^{k}}\right)$.

Proof. It suffices to check that the result holds for $\xi \times 1: E \times D^{s} \rightarrow M \times D^{s}, s$ large. If $\xi^{\#} \neq 0$ (the hypotheses of Lemma C16(a) are satisfied for $\xi \oplus 1_{s}$ and the trivial bundle), we can construct (by $\mathrm{Cl6}(\mathrm{a})$ ) $\phi: Y \rightarrow M$, embed $Y$ in $M \times D^{s}$ and take $N$ to be a regular neighborhood. Then $\xi \times\left. 1_{D^{s}}\right|_{N}$ is stably fibre homotopically trivial. Then by $\mathrm{C} 11$ and $\mathrm{C} 15$ the result holds for $\xi \times 1_{D^{s}} \mid N$. Now by the remark before C13, $i: \pi_{j} C(N) \rightarrow \pi_{j} C\left(M \times D^{s}\right)$ corresponds to $\pi_{j}(P(Y)) \rightarrow^{P(\phi)} \pi_{j}(P(M))$. But $P(\phi)$ is a rational $r$ equivalence provided $\phi$ is, by the relation between $P(X)$ and Waldhausen's functor $A(X)$ [W]. Thus for $s$ sufficiently large $i: C(N) \rightarrow$ $C\left(M \times D^{s}\right)$ will be a rational $\omega(n)$ equivalence. Since $i$ commutes with $T\left(\varepsilon \oplus 1_{s}\right)$, hence also with $\theta\left(\xi \oplus 1_{s}\right)$, the result holds for $\xi \oplus 1_{s}$. Since $T$ and hence $\theta$ commutes with $\Sigma^{s}$, the result holds for $\xi$. If $\xi^{\#}=0$ we can extend $\xi \oplus 1_{s}$ to $\hat{\xi}$ over $M \times S^{2} \times D^{s-2}$ (by $\mathrm{Cl}$ (b)) so that $\hat{\xi}$ restricts to $\xi \oplus 1_{s}$ on

$$
M \times D^{2} \times D^{s-2}\left(M \times D^{2} \times D^{s-2} \subset M \times S^{2} \times D^{s-2}\right) \text {. }
$$


We prove the statement for $\hat{\xi}$ instead of $\xi \oplus 1_{s}$ which implies the statement for $\xi \oplus 1_{s}$ because of the naturality of $T(\xi)$ or $\theta(\xi)$ (by A2) and because the composition

$$
C\left(M \times D^{2} \times D^{s-2}\right) \hookrightarrow C\left(M \times S^{2} \times D^{s-2}\right) \subset C\left(M \times D^{s}\right)
$$

is a homotopy equivalence.

THEOREM C18. Let $\xi: E \rightarrow M^{n}$ be a smooth orthogonal or topological $S^{2 k}$ bundle, $k$ large. Then on the $\omega(n)$ Postnikov term and localized at zero, $T: C(M) \rightarrow C(E)$ is an equivalence.

Proof. It suffices to check that the result holds for the bundle $\xi \times 1_{D^{s}}$ : $E \times D^{s} \rightarrow M \times D^{s}$, s large. If $\xi^{\#} \neq 0$ let $\phi: Y \rightarrow M$ be as in C16(a) (for $\xi \oplus 1_{s}$ and the trivial bundle), embed $Y$ in $M \times D^{s}$ and let $N$ be a regular neighborhood. Then $\left(\xi \times 1_{D^{s}}\right) \mid N$ is fibre homotopically trivial. By $\mathrm{ClO}(\mathrm{d})$ and $\mathrm{Cl}$, the result holds for $\left(\xi \times 1_{D^{s}}\right) \mid N$. Now by the remarks before C13, $i: \pi_{j} C(N) \rightarrow \pi_{j} C\left(M \times D^{s}\right)$ corresponds to $P(\phi): \pi_{j} P(Y) \rightarrow \pi_{j} P(M)$. But rationally, $P(\phi)$ is highly connected provided $\phi$ is, by the relation between $P(X)$ and Waldhausen's functor $A(X)$ [W]. Thus for $s$ sufficiently large $i: C(N) \rightarrow C\left(M \times D^{s}\right)$ will be a rational $\omega(n)$ equivalence. Since $T$ commutes with $i$, the result holds for $\xi \oplus 1_{s}$ and hence for $\xi$. If $\xi^{\#}=0$ we reduce to the case $\xi^{\#} \neq 0$ by extending $\xi$ to $\hat{\xi}$ as in Theorem C17.

\section{The functor $\delta$.}

Definition of $\delta$. For $X \in \mathbf{P}$, let $N^{n}(X)$ be a regular neighborhood of $X$ embedded in some large Euclidean space $E^{n}$. For $n$ sufficiently large, $N^{n}(X)$ is unique up to isomorphism. Further, $N^{n+1}(X)=N^{n}(X) \times I$. Define $\delta(X)$ $=\lim _{n} \tilde{C}\left(N^{n}(X)\right) / C\left(N^{n}(X)\right)$, where the limit is taken under $\Sigma$ : $\tilde{C}\left(\vec{N}^{n}(X)\right) / C\left(N^{n}(X)\right) \rightarrow \tilde{C}\left(N^{n+1}(X)\right) / C\left(N^{n+1}(X)\right)$.

If $f: X \rightarrow Y$, then $f$ defines a unique homotopy class of maps of $N^{n}(X)$ into $N^{n}(Y)$. For $m \gg n$, this gives a well-defined isotopy class of embeddings of $N^{n}(X)$ in $N^{m}(Y)$ with trivial normal bundle and hence an isotopy class of embeddings $f_{m}$ : $N^{m}(X)=N^{n}(X) \times D^{m-n} \rightarrow N^{m}(Y)$. Further, if we take $f_{m+1}=f_{m} \times$ id,

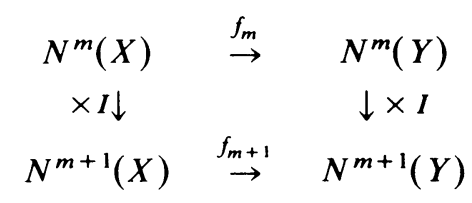

commutes, and

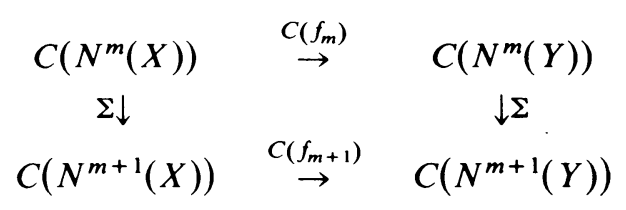

commutes, and similarly for $\tilde{C}$. Thus $f$ defines a map $\delta(f): \delta(X) \rightarrow \delta(Y)$ such that $f \sim g$ implies $\delta(f)=\delta(g)$. 
Connectivity of $\delta$.

Proposition D1. If $f: X \rightarrow Y$ is $r$-connected and $Y$ is $k$-connected, then $\delta(f)$ : $\delta(X) \rightarrow \delta(Y)$ is $\inf (2 r-2, r+k-1)$ - (resp. $\inf (2 r-2, r+k-1, r+3)$-) connected in the Diff (resp. Top) category.

Proof. For $n$ sufficiently large, we can assume $N^{n}(Y)$ is built up from $N^{n}(X)$ by adding handles of index $>r$, and that the inclusion of $N^{n}(X)$ in $N^{n}(Y)$ corresponds to $f$. By Proposition Bl,

$$
\tilde{C} / C\left(N^{n}(X)\right) \rightarrow \tilde{C} / C\left(N^{n}(Y)\right)
$$

is $\inf (2 r-2, r+k-1)$ - (resp. $\inf (2 r-2, r+k-1, r+3)$-) connected in the Diff (resp. Top) category and the result follows.

Corollary D2. If $f: X \rightarrow Y$ is $r$-connected, $\delta(f): \delta(X) \rightarrow \delta(Y)$ is $(r-1)$ connected.

PROPOSITION D3. If for the diagram

$$
\begin{array}{ccc}
A \cap B & \rightarrow & B \\
f \downarrow & & \downarrow g \\
A & \rightarrow & A \cup B
\end{array} \text { in } \mathbf{P}
$$

the horizontal inclusions are $p$-connected and the vertical inclusions are $q$-connected, $p, q \geqslant 2$, then the induced map, Homotopy fibre $\delta(f) \rightarrow$ Homotopy fibre $\delta(g)$, is $(p+q-3)$-connected $\left((p+q-2)\right.$-connected if $\left.\pi_{1}(A \cup B)=0\right)$.

Proof. Let $N^{n}(A \cap B)$ be a regular neighborhood in $E^{n}, n$ large. We may add handles of index $>q$ to $N^{n}(A \cup B)$ to build up $N^{n}(A)$ and handles of index $>p$ to build up $N^{n}(B)$, so that $N^{n}(A) \cap N^{n}(B)=N^{n}(A \cap B)$ and $N^{n}(A) \cup N^{n}(B)=$ $N^{n}(A \cup B)$. Dually, this means (1) we may add handles of index $<n-q$ to $\partial N^{n}(A) \times I$ in $N^{n}(A)$ to build up a manifold $V^{n}$ such that

$$
N^{n}(A)-\stackrel{\circ}{V}=N^{n}(A \cap B) \text { and } N^{n}(A \cup B)-\dot{V}=N^{n}(B) \text {, }
$$

and (2) we may add handles of index $<n-p$ to $\partial N^{n}(B) \times I$ in $N^{n}(B)$ to build up a manifold $W^{n}$ such that

$$
N^{n}(B)-\stackrel{\circ}{W}=N^{n}(A \cap B) \text { and } N^{n}(A \cup B)-\stackrel{\circ}{W}=N^{n}(A) .
$$

Thus we have a map of fibrations

$$
\begin{aligned}
& C\left(N^{n}(A \cap B)\right) \quad \rightarrow \quad C\left(N^{n}(A)\right) \quad \rightarrow \quad C \operatorname{Emb}\left(V, N^{n}(A)\right)
\end{aligned}
$$

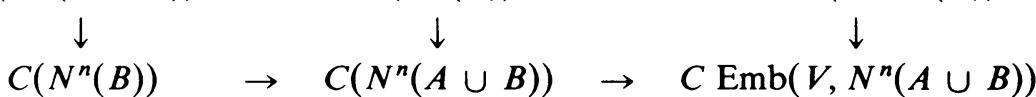

Now $C \operatorname{Emb}\left(V, N^{n}(A)\right)=C \operatorname{Emb}\left(V, N^{n}(A \cup B)-\dot{W}\right)$. By Morlet's lemma of disjunction [BLR], applied inductively over the handles of $V$ and $W$, $\pi_{i}\left(C \operatorname{Emb}\left(V, N^{n}(A \cup B)\right)\right), C \operatorname{Emb}\left(V, N^{n}(A \cup B)-W\right)=0$ for $i<p+q-3$, $p \geqslant 2, q \geqslant 2\left(i \leqslant p+q-2\right.$ if $\left.\pi_{1}(A \cup B)=0\right)$. Thus,

Homotopy fibre $\left(B C\left(N^{n}(A \cap B)\right)\right) \rightarrow B C\left(N^{n}(A)\right)$

$$
\rightarrow \text { Homotopy fibre }\left(B C\left(N^{n}(B)\right)\right) \rightarrow B C\left(N^{n}(A \cup B)\right)
$$

will be $(p+q-3)$-connected $\left((p+q-2)\right.$-connected if $\left.\pi_{1}(A \cup B)=0\right)$. Since $\delta(X)=\lim _{\rightarrow} \tilde{C} / C\left(N^{n}(X)\right)=\lim _{\rightarrow_{n}} B C\left(N^{n}(X)\right)$, the result follows. 
Proposition D4. If $f: X \rightarrow Y$ is a rational homotopy equivalence and $f_{1}: \pi_{1}(X) \simeq$ $\pi_{1}(Y)$, then $\delta(f): \mathcal{S}(X) \rightarrow \mathcal{S}(Y)$ is a rational homotopy equivalence.

Proof. Following Waldhausen [W], $\delta(X)=\Omega W h(X)$ and one has the fibration, $h(X, A(*)) \rightarrow A(X) \rightarrow W h^{\mathrm{PL}}(X)$, where $h(X, A(*))$ is a homology theory. Waldhausen proves for his functor $A$ that if $\Omega f: \Omega X \rightarrow \Omega Y$ is a rational homotopy equivalence so is $A(f): A(X) \rightarrow A(Y)$ (see proof of Proposition 1.1 of [W]). This implies the result for $\mathcal{S}^{\mathrm{PL}}(X)$ and hence for $\mathcal{S}^{\mathrm{Top}}(X)$. The result for $\mathcal{S}^{\text {Diff }}$ follows from the fibration (see [W] or [BL]) $\delta^{\text {Diff }}(X) \rightarrow \delta^{\mathrm{PL}}(X) \rightarrow \delta_{S}^{\mathrm{PL}}(X)$, where $\delta_{S}^{\mathrm{PL}}(X)$ is the stabilization of $\mathcal{S}^{\mathrm{PL}}(X)$ under suspension, $\delta_{S}^{\mathrm{PL}}(X)$ being a homology theory.

REMARK. One may also prove D2 and D3 via Waldhausen which in turn proves the corresponding result at the concordance group level in the stable range. For D4, however, it seems unlikely that a proof starting at the concordance level will be possible.

Definition of $g(X)$. $\delta\left(X \times S^{1}\right) \rightarrow B \delta(X)$.

Consider the principal fibration [BLR],

$$
C(M \times I) \rightarrow C(M \times[0, \infty)) \rightarrow C \operatorname{Emb}(M, M \times R) .
$$

Since $C(M \times[0, \infty))$ is clearly contractible, the classifying map

$$
C \operatorname{Emb}(M, M \times R) \rightarrow B C(M \times I)
$$

is a homotopy equivalence. On the other hand we have the map $\lambda: C\left(M \times S^{1}\right) \rightarrow$ $C(M \times R)$ obtained by taking the unique lift of a concordance of $M \times S^{1}$ to the covering space $M \times R$. Thus we get a map, $\gamma(M): C\left(M \times S^{1}\right) \rightarrow C(M \times R) \rightarrow$ $C \operatorname{Emb}(M, M \times R) \rightarrow B C(M \times I)$.

Now if $X \in \mathbf{P}, N^{n+1}\left(X \times S^{1}\right)=N^{n-1}(X) \times S^{1} \times I=N^{n}(X) \times S^{1}, n$ large. Define $g_{n}(X)=\gamma\left(N^{n}(X)\right): \quad C\left(N^{n+1}\left(X \times S^{1}\right)\right)=C\left(N^{n}(X) \times S^{1}\right) \rightarrow$ $B C\left(N^{n+1}(X)\right)$. We need to show $g_{n}(X)$ commutes with $\Sigma$. One can define $\Sigma$ : $C \operatorname{Emb}(M, M \times R) \rightarrow C \operatorname{Emb}(M \times I, M \times I \times R)$ or more generally, given $L^{k}$ properly embedded in $N^{n}, \xi: E \rightarrow N$ an $\mathcal{Q}$ bundle, one can define $T(\xi)$ : $C \operatorname{Emb}\left(L^{k}, N^{n}\right) \rightarrow C \operatorname{Emb}\left(\xi^{-1}(L), E\right)$ as in $\S \mathrm{A}$. In particular, we obtain a commutative diagram

$$
\begin{array}{ccccc}
C(M \times I) & \rightarrow & C(M \times[0, \infty)) & \rightarrow & C \operatorname{Emb}(M, M \times R) \\
\Sigma \downarrow & & \Sigma \downarrow & & \\
C\left(M \times I^{2}\right) & \rightarrow & C(M \times I \times[0, \infty)) & \rightarrow & C \operatorname{Emb}(M \times I, M \times I \times R)
\end{array}
$$

and hence a commutative diagram

$$
\begin{array}{ccc}
C \operatorname{Emb}(M, M \times R) & \rightarrow & B C(M \times I) \\
\Sigma \downarrow & & \downarrow B \Sigma \\
C \operatorname{Emb}(M \times I, M \times I \times R) & \rightarrow & B C\left(M \times I^{2}\right)
\end{array}
$$

Further, if $M=N \times I^{r}$, the diagram deloops $r$ times, and so does the map $\gamma(M)$. Likewise all these results hold for $\tilde{C}$. Thus $g_{n}(X): \tilde{C} / C\left(N^{n+1}\left(X \times S^{1}\right)\right) \rightarrow$ $B \tilde{C} / C\left(N^{n+1}(X)\right)$ is defined for $n$ sufficiently large, and commutes with $\Sigma$. Define $g(X)=\lim _{n} g_{n}(X)$. Note that $g$ is natural with respect to $X$. 
The $W K$ property. Let Nil $\delta(X)=$ Fibre $g(X) \times \delta\left(p_{X}\right): \delta\left(X \times S^{1}\right) \rightarrow B \delta(X)$ $\times \delta(X)$. Then Nil $\delta$ is a functor and satisfies D2-D4.

Now consider the commutative diagram:

$$
\begin{aligned}
& C(M \times I) \stackrel{i}{\rightarrow} C\left(M \times S^{1}\right) \stackrel{r}{\rightarrow} C \operatorname{Emb}\left(M \times M \times S^{1}\right) \\
& \lambda \downarrow \\
& \downarrow \lambda \\
& C(M \times R) \stackrel{\simeq}{\rightarrow} C \operatorname{Emb}(M, M \times R) \stackrel{\simeq}{\rightarrow} B C(M \times I)
\end{aligned}
$$

where the top row is a fibration. In [BLR] it is shown that

$$
\lambda: C\left(M \times S^{1}\right) \rightarrow C(M \times R)
$$

has a right inverse $\mu$, and if $M=N \times I, i$ has a left inverse $j=j(M): C\left(M \times S^{1}\right)$ $\rightarrow C(M \times I)$ induced by the inclusion $j: N \times I \times S^{1} \rightarrow N \times I^{2}$. Thus if $M=N \times I$ the fibration $r$ is trivial, and if we let $C \Re(M)$ be the fibre of $\lambda$ : $C \operatorname{Emb}\left(M, M \times S^{1}\right) \rightarrow C \operatorname{Emb}(M, M \times R), C\left(M \times S^{1}\right) \sim C(M \times I) \times$ $B C(M \times I) \times C \Re(M)$.

For $n$ sufficiently large and $j_{n}(X)=j\left(N^{n}(X)\right)$,

$$
g_{n}(X) \times j_{n}(X): C\left(N^{n+1}\left(X \times S^{1}\right)\right) \rightarrow B C\left(N^{n+1}(X)\right) \times C\left(N^{n+1}(X \times I)\right)
$$

is a trivial fibration with fibre $C \Re\left(N^{n}(X)\right)$. The same results hold for $\tilde{C}$. Since $\lim _{n} j_{n}(X)=\delta\left(p_{X}\right)$

$$
j_{n}(X): \tilde{C} / C\left(N^{n+1}\left(X \times S^{1}\right)\right) \rightarrow \tilde{C} / C\left(N^{n+1}(X \times I)\right),
$$

$g(X) \times \delta\left(p_{X}\right)$ is a trivial fibration.

REMARK. Since $\tilde{C}()$ is contractible, Nil $\delta(X)=\lim _{\rightarrow} B C \mathscr{N}\left(N^{n}(X)\right)$ and $\Omega$ Nil $\delta(X) \stackrel{\omega(n)}{\sim} C \Re\left(N^{n}(X)\right)$.

DEFINITION OF $Q$ TRANSFER. Let $\xi: E \rightarrow X$ be a locally trivial bundle with fibre $F$ a compact $Q$ manifold and structure group $\underline{A}^{\mathbb{Q}}(F)$. If $N^{n}(X)$ is a regular neighborhood of $X$ in $E^{n}, X \subset N^{n}(X) \rightarrow X$, then $r_{n}^{*} \xi: E\left(r_{n}^{*} \xi\right) \rightarrow N^{n}(X)$ is a locally trivial $\mathbb{Q}$ bundle. If $\nu_{n}^{s}$ is a normal $\mathbb{Q}$ disc bundle of $E\left(r_{n}^{*} \xi\right)$ in $E^{s}, s$ large, then $E\left(\nu_{n}^{s}\right)$ is a regular neighborhood $N^{s}(E)$ of $E$. Since $E\left(r_{n+1}^{*} \xi\right)=E\left(r_{n}^{*} \xi\right) \times I$, we can take $E\left(\nu_{n+1}^{s+1}\right)=E\left(\nu_{n}^{s}\right) \times I=N^{s+1}(E)$. Then

$$
\begin{array}{ccccc}
C\left(N^{n}(X)\right) & \underset{T\left(r_{n}^{*} \xi\right)}{\rightarrow} & C\left(E\left(r_{n}^{*} \varepsilon\right)\right) & \underset{T\left(\nu_{n}^{s}\right)}{\rightarrow} & C N^{s}(E) \\
\Sigma \downarrow & & \Sigma \downarrow & & \Sigma \downarrow \\
C\left(N^{n+1}(X)\right) & \underset{T\left(r_{n+1}^{*} \xi\right)}{\rightarrow} & C\left(E\left(r_{n+1}^{*} \varepsilon\right)\right) & \underset{T\left(\nu_{n}^{s+1}\right)}{\rightarrow} & C N^{s+1}(E)
\end{array}
$$

commutes. Similarly, for $\tilde{C}$. Thus define $T(\varepsilon): \quad \delta(X) \rightarrow \delta(E)$ as $T(\xi)=$ $\lim _{k} T\left(\nu_{n+k}^{s+k}\right) \circ T\left(r_{n+k}^{*} \xi\right), T$ defined on $\tilde{C} / C()$.

Notation. We extend the term $\mathbb{Q}$ bundle to include bundles $\xi$ over $X \in \mathbf{P}$ as above.

Proposition D5. If $E_{1} \stackrel{\xi_{1}}{\rightarrow} E_{2} \stackrel{\xi_{2}}{\rightarrow} X$ is a composition of $\mathbb{Q}$ bundles, $T^{\mathcal{Q}}\left(\xi_{2} \circ \xi_{1}\right)=$ $T^{\mathbb{Q}}\left(\xi_{1}\right) \circ T^{\mathbb{Q}}\left(\xi_{2}\right)$ on $\delta^{\mathbb{Q}}(X)$. 
Proof. Since

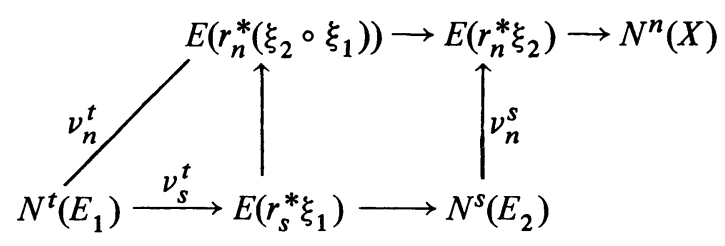

commutes, by Proposition Al,

$$
T\left(\nu_{n}^{t}\right) \circ T\left(r_{n}^{*}\left(\xi_{2} \circ \xi_{1}\right)\right)=T\left(\nu_{s}^{t}\right) \circ T\left(r_{s}^{*} \xi_{1}\right) \circ T\left(\nu_{n}^{s}\right) \circ T\left(r_{n}^{*} \xi_{2}\right),
$$

and the result is immediate.

Proposition D6. If

$$
\begin{array}{ccc}
E_{1} & \stackrel{f_{E}}{\rightarrow} & E_{2} \\
\xi_{1} \downarrow & & \downarrow \xi_{2} \\
X_{1} & \stackrel{f_{X}}{\rightarrow} & X_{2}
\end{array}
$$

is a cartesian diagram of $\mathcal{Q}$ bundles then $T\left(\xi_{2}\right)\left(f_{X}\right)=\delta\left(f_{E}\right) T\left(\xi_{1}\right)$.

Proof. For $n$ sufficiently large, $f_{X}$ defines an isotopy class of embeddings $\left(f_{X}\right)_{n}$ of $N^{n}\left(X_{1}\right)$ in $N^{n}\left(X_{2}\right)$, and

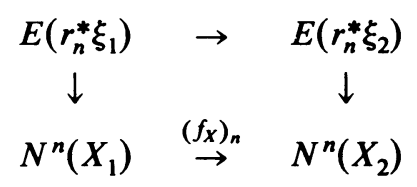

is cartesian, where the horizontal arrow at the top is an embedding. Thus for $s$ sufficiently large

$$
\begin{array}{ccc}
N^{s}\left(E_{1}\right) & \stackrel{\left(f_{E}\right)_{s}}{\rightarrow} & N^{s}\left(E_{2}\right) \\
r_{n}^{*} \xi_{1} \circ \nu_{n}^{s} \downarrow & & \downarrow r_{n}^{*} \xi_{2} \circ \nu_{n}^{s} \\
N^{n}\left(X_{1}\right) & \stackrel{\left(f_{x}\right)_{n}}{\rightarrow} & N^{n}\left(X_{2}\right)
\end{array}
$$

is cartesian, and the result follows from Proposition A2.

Proposition D7. If $\varepsilon_{K}$ is the trivial $Q$ bundle over $X$ with fibre $K$ and $s:$ $X \rightarrow X \times K$ is the cross-section $s(x)=\left(x, k_{0}\right)$, then

$$
T\left(\varepsilon_{K}\right)=\chi(K) \cdot \delta(s)
$$

Proof. If $N^{m}(K)$ is the normal disc bundle of $K$ in $E^{m}$ and $N^{n}(X)$ is a regular neighborhood of $X$ in $E^{n}$, then $N^{n}(X) \times N^{m}(K)=N^{n+m}(X \times K)$ is a regular neighborhood of $X \times K$ in $E^{n+m}$. Let $N_{n}^{n+m}\left(\varepsilon_{K}\right): N^{n}(X) \times N^{m}(K) \rightarrow N^{n}(X)$ be the trivial bundle, and let $D^{m}=N^{m}\left(k_{0}\right) \subset N^{m}(K)$. Then by Proposition A4, $T\left(N_{n}^{n+m}\left(\varepsilon_{K}\right)\right)=\left(i \Sigma^{m}\right)^{\chi\left(N^{m}(K)\right)}$. But $\chi\left(N^{m}(K)\right)=\chi(K)$, and $i \Sigma^{m}: \quad C\left(N^{m}(X)\right) \rightarrow$ $C\left(N^{n}(X) \times D^{m}\right) \rightarrow C\left(N^{n}(X) \times N^{m}(K)\right)$ is the map induced by the embedding $s_{m}$ : $N^{n+m}(X)=N^{n}(X) \times D^{m} \rightarrow N^{n+m}(X \times K)$, corresponding to $s$. Hence $T\left(\varepsilon_{K}\right)=$ $\xi(K) \cdot \delta(s)$ 
Corollary D8. $\delta\left(\varepsilon_{K}\right) T\left(\varepsilon_{K}\right)=\chi(K) \cdot 1_{\delta(X)}$.

Proposition D9. $T$ is compatible with $g$.

Just as for $\Sigma$ (see WK property above) one has for $\xi: E \rightarrow M$ the commutative diagram

$$
\begin{array}{ccc}
C \operatorname{Emb}(M, M \times R) & \rightarrow & B C(M \times I) \\
T\left(\xi \times \mathrm{id}_{R}\right) \downarrow & & \downarrow B T\left(\xi \times \mathrm{id}_{I}\right) \\
C \operatorname{Emb}(E, E \times R) & \rightarrow & B C(E \times I)
\end{array}
$$

from which one gets the commutative diagram

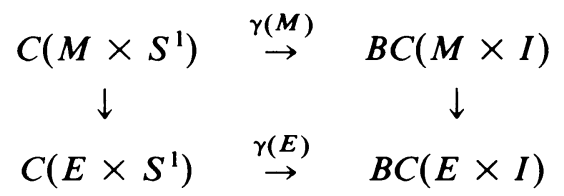

where the left and right vertical maps are $T\left(\xi \times \mathrm{id}_{S^{\prime}}\right)$ and $B T\left(\xi \times \mathrm{id}_{I}\right)$ respectively. Now let $\xi: E \rightarrow X$. Then

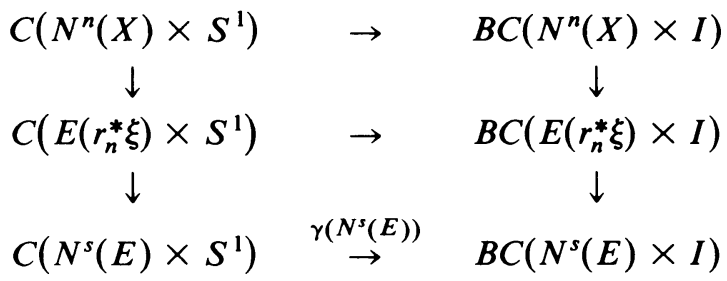

commutes where the left vertical maps are $T\left(r_{n}^{*} \xi \times \mathrm{id}_{S^{1}}\right)$ and $T\left(\nu_{n}^{s} \times \mathrm{id}_{s^{1}}\right)$; the right vertical maps $B T\left(r_{n}^{*} \xi \times \mathrm{id}_{I}\right)$ and $B T\left(\nu_{n}^{s} \times \mathrm{id}_{I}\right)$, and the horizontal maps $\gamma\left(N^{n}(x)\right), \gamma\left(E\left(r_{n}^{*} \xi\right)\right)$ and $\gamma\left(N^{s}(E)\right)$. Since $\gamma\left(N^{n}(X)\right)=g_{n}(X), \gamma\left(N^{s}(E)\right)=g_{s}(E)$, and $r_{n+1}^{*}\left(\xi \times \mathrm{id}_{S^{1}}\right)=r_{n}^{*} \xi \times \mathrm{id}_{S^{1}}, \nu_{n+1}^{s+1}=\nu_{n}^{s} \times \mathrm{id}_{S^{1}}$, we get in the limit:

$$
g(E) \circ T\left(\xi \times \mathrm{id}_{S^{1}}\right)=B T(\xi) g(X) \text { on } \delta\left(X \times S^{1}\right) \text {. }
$$

DeFINITION OF THE $\mathcal{Q}$-INVOLUTION FOR $\mathcal{\delta}$. If $\alpha$ is an orthogonal or topological $k$ disc bundle over $X$, denote the involution $\tau$ on $C\left(E\left(r_{n}^{*} \alpha\right)\right)$ by $\tau_{n}^{\alpha}$. Since $T\left(r_{n}^{*} \alpha\right)$ : $C\left(N^{n}(X)\right) \rightarrow C\left(E\left(r_{n}^{*} \alpha\right)\right)$ is an $\omega(n)$ equivalence by $C 10(\mathrm{a})$, we can define an involution on $\left[C\left(N^{n}(X)\right)\right]_{\omega(n)}$ by $T\left(r_{n}^{*} \alpha\right)^{-1} \tau_{n}^{\alpha} T\left(r_{n}^{*} \alpha\right)$. If we replace $\alpha$ by $\alpha \oplus 1$, then

$$
\begin{aligned}
T\left(r_{n}^{*}(\alpha \oplus 1)\right)^{-1} \tau_{n}^{\alpha \oplus 1} T\left(r_{n}^{*}(\alpha \oplus 1)\right) & =T\left(r_{n}^{*} \alpha\right)^{-1} \Sigma^{-1} \tau_{n}^{\alpha} \Sigma T\left(r_{n}^{*} \alpha\right) \\
& =-T\left(r_{n}^{*} \alpha\right)^{-1} \tau_{n}^{\alpha} T\left(r_{n}^{*} \alpha\right) .
\end{aligned}
$$

If we replace $N^{n}(X)$ by $N^{n+1}(X)$, then $E\left(r_{n+1}^{*} \alpha\right)=E\left(r_{n}^{*} \alpha\right) \times I$ and $T\left(r_{n+1}^{*} \alpha\right)^{-1} \tau_{n+1}^{\alpha} T\left(r_{n+1}^{*} \alpha\right) \Sigma=T\left(r_{n+1}^{*} \alpha\right)^{-1} \tau_{n+1}^{\alpha} \Sigma T\left(r_{n}^{*} \alpha\right)=-T\left(r_{n+1}^{*} \alpha\right)^{-1} \Sigma \tau_{n}^{\alpha} T\left(r_{n}^{*} \alpha\right)$ $=-T\left(r_{n}^{*} \alpha\right)^{-1} \tau_{n}^{\alpha} T\left(r_{n}^{*} \alpha\right)$. Let $\hat{\tau}_{n}^{\alpha}=(-1)^{n+k} T\left(r_{n}^{*} \alpha\right)^{-1} \tau_{n}^{\alpha} T\left(r_{n}^{*} \alpha\right)$. Define $\tau^{\alpha}$ on $\delta(X)=\lim _{\rightarrow} \tilde{C} / C\left(N^{n}(X)\right)$ by $\tau^{\alpha}=\lim _{\rightarrow} \hat{\tau}_{n}^{\alpha}$. Then $\tau^{\alpha}$ depends only on $\alpha$ as a stable (vector or micro) bundle.

Proposition D10. If $\alpha \in \tilde{K}(Y)$ and $f: X \rightarrow Y$, then $\delta(f):\left(\delta(X), \tau^{f^{*} \alpha}\right) \rightarrow$ $\left(\mathcal{S}(Y), \tau^{\alpha}\right)$ is equivariant. 
Proof. We have the homotopy commutative diagram

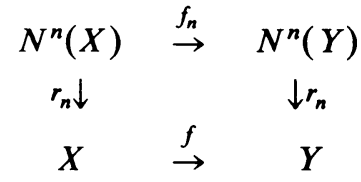

Hence $r_{n}^{*} f^{*} \alpha \sim f_{n}^{*} r_{n}^{*} \alpha$. If $E\left(f_{n}\right): E\left(r_{n}^{*} f^{*} \alpha\right) \rightarrow E\left(r_{n}^{*} \alpha\right)$ is the bundle map covering the embedding $f_{n}, C\left(E\left(f_{n}\right)\right) T\left(r_{n}^{*} f^{*} \alpha\right)=T\left(r_{n}^{*} \alpha\right) C\left(f_{n}\right)$ and letting $\beta_{n}=f_{n}^{*} \alpha$,

$$
C\left(E\left(f_{n}\right)\right) \tau_{n}^{\beta_{n}}=\tau_{n}^{\alpha} C\left(E\left(f_{n}\right)\right) .
$$

Hence

$$
\begin{aligned}
C\left(f_{n}\right) T\left(r_{n}^{*} f^{*} \alpha\right)^{-1} \tau_{n}^{\beta_{n}} T & \left(r_{n}^{*} f^{*} \alpha\right)=T\left(r_{n}^{*} \alpha\right)^{-1} C\left(E\left(f_{n}\right)\right) \tau_{n}^{\beta_{n}} T\left(r_{n}^{*} f^{*} \alpha\right) \\
& =T\left(r_{n}^{*} \alpha\right)^{-1} \tau_{n}^{\alpha} C\left(E\left(f_{n}\right)\right) T\left(r_{n}^{*} f^{*} \alpha\right)=T\left(r_{n}^{*} \alpha\right)^{-1} \tau_{n}^{\alpha} T\left(r_{n}^{*} \alpha\right) C\left(f_{n}\right) .
\end{aligned}
$$

Thus $C\left(f_{n}\right) \hat{\tau}_{n}^{\beta_{n}}=\hat{\tau}_{n}^{\alpha} C\left(f_{n}\right)$. The result follows by passage to the limit.

Proposition D10(2). Let $\alpha$ be an orthogonal or topological $k$ disc bundle over $X$. Then $g(X):\left(\delta\left(X \times S^{1}\right), \tau^{\beta}\right) \rightarrow\left(B S(X), B \tau^{\alpha}\right)$ is equivariant, where $\beta=p_{X}^{*} \alpha$.

Proof. Since $r_{n+1}: N^{n+1}\left(X \times S^{1}\right)=N^{n}(X) \times S^{1} \rightarrow X \times S^{1}$ is $r_{n} \times 1_{S^{1}}$, where $r_{n}: N^{n}(X) \rightarrow X$, we have $p_{X} \circ r_{n+1}=r_{n} \circ p_{N^{n}(X)}$ and $E\left(r_{n+1}^{*} \beta\right)=E\left(r_{n}^{*} \alpha\right) \times S^{1}$. Now (see D9), $B T\left(r_{n+1}^{*} \alpha\right) \circ \gamma\left(E\left(r_{n}^{*} \alpha\right)\right)=\gamma\left(E\left(r_{n}^{*} \alpha\right)\right) \circ T\left(r_{n+1}^{*} \beta\right)$. Also $\gamma\left(E\left(r_{n}^{*} \alpha\right)\right) \tau_{n+1}^{\beta} \sim B\left(\tau_{n+1}^{\alpha}\right) \gamma\left(E\left(r_{n}^{*} \alpha\right)\right)$. In fact, $C\left(E\left(r_{n}^{*} \alpha\right) \times S^{1}\right) \rightarrow C\left(E\left(r_{n}^{*} \alpha\right) \times R\right) \rightarrow$ $\Omega B C\left(E\left(r_{n}^{*} \alpha\right) \times R\right)$ is equivariant with respect to $\tau$ since by May's recognition principle $C\left(E\left(r_{n}^{*} \alpha\right) \times R\right) \rightarrow \Omega B C\left(E\left(r_{n}^{*} \alpha\right) \times R\right)$ is a natural equivalence [M]; and $B \bar{\phi}: B C\left(E\left(r_{n}^{*} \alpha\right) \times I\right) \rightarrow B \Omega C\left(E\left(r_{n}^{*} \alpha\right) \times R\right) \rightarrow \Omega B C\left(E\left(r_{n}^{*} \alpha\right) \times R\right)$ is an equivariant homotopy equivalence by $\S \mathrm{B}$, addendum.

Thus $g_{n}(X) \circ \tau_{n+1}^{\beta} \sim B\left(\hat{\tau}_{n+1}^{\alpha}\right) \circ g_{n}(X)$, and $g(X)$ is equivariant.

Homotopy invariance of the involutions. Suppose $\alpha, \beta \in \tilde{K}_{\mathbb{Q}}(X)$ are stably fibre homotopy equivalent. Then $\beta-\alpha$ is represented by a bundle which is stably fibre homotopically trivial. Let

$$
r_{n}^{*} \alpha: E\left(r_{n}^{*} \alpha\right) \rightarrow N^{n}(X)
$$

be represented by a $p$ disc bundle, and $\beta-\alpha$ by a $q$ disc bundle. Then $r_{n}^{*} \beta$ is represented by $r_{n}^{*} \alpha \oplus r_{n}^{*}(\beta-\alpha)$, a $p+q$ disc bundle. Let $\delta_{n}$ be $\beta-\alpha$ pulled back over $E\left(r_{n}^{*} \alpha\right)$. Then $r_{n}^{*} \beta=\delta_{n} \circ r_{n}^{*} \alpha$. Since $\delta_{n}$ is stably fibre homotopically trivial, we have in the Top category (by C11): $T\left(\delta_{n}\right) \tau_{n}^{\alpha}=(-1)^{q} \tau_{n}^{\beta} T\left(\delta_{n}\right)$. Hence

$$
\begin{aligned}
(-1)^{p} T\left(r_{n}^{*} \alpha\right)^{-1} \tau_{n}^{\alpha} T\left(r_{n}^{*} \alpha\right) & =(-1)^{p} T\left(r_{n}^{*} \alpha\right)^{-1} T\left(\delta_{n}\right)^{-1} T\left(\delta_{n}\right) \tau_{n}^{\alpha} T\left(r_{n}^{*} \alpha\right) \\
& =(-1)^{p+q} T\left(r_{n}^{*} \alpha\right)^{-1} T\left(\delta_{n}\right)^{-1} \tau_{n}^{\beta} T\left(\delta_{n}\right) T\left(r_{n}^{*} \alpha\right) \\
& =(-1)^{p+q} T\left(r_{n}^{*} \beta\right)^{-1} \tau_{n}^{\beta} T\left(r_{n}^{*} \beta\right) .
\end{aligned}
$$

So $\hat{\tau}_{n}^{\alpha}=\hat{\tau}_{n}^{\beta}$ and $\tau^{\alpha}=\tau^{\beta}$ on $\delta(X)$. This proves

Proposition D11. If $\alpha, \beta \in \tilde{K}_{\text {Top }}(X)$ are stably fibre homotopy equivalent, then $\tau^{\alpha}=\tau^{\beta}$ on $\delta^{\text {Top }}(X)$. 
In the Diff category, by $\mathrm{C} 15$ there is an $\omega(n)$ equivalence localized away from two, $\theta\left(\delta_{n}\right): C\left(E\left(r_{n}^{*} \alpha\right)\right) \rightarrow C\left(E\left(\delta_{n} \circ r_{n}^{*} \alpha\right)\right)$ such that $\theta\left(\delta_{n}\right) \tau_{n}^{\alpha}=(-1)^{q} \tau_{n}^{\beta} \theta\left(\delta_{n}\right)$. Thus localized away from two,

$$
(-1)^{p} T\left(r_{n}^{*} \alpha\right)^{-1} \tau_{n}^{\alpha} T\left(r_{n}^{*} \alpha\right)=(-1)^{p+q} T\left(r_{n}^{*} \alpha\right)^{-1} \theta\left(\delta_{n}\right)^{-1} \tau_{n}^{\beta} \theta\left(\delta_{n}\right) T\left(r_{n}^{*} \alpha\right) .
$$

Define $\theta(X, \alpha, \beta)=\lim _{n} T\left(r_{n}^{*} \beta\right)^{-1} \theta\left(\delta_{n}\right) T\left(r_{n}^{*} \alpha\right)$. Since

$$
T\left(r_{n}^{*} \beta\right)^{-1} \theta\left(\delta_{n}\right) T\left(r_{n}^{*} \alpha\right) \hat{\tau}_{n}^{\alpha}=\hat{\tau}_{n}^{\beta} T\left(r_{n}^{*} \beta\right)^{-1} \theta\left(\delta_{n}\right) T\left(r_{n}^{*} \alpha\right)
$$

$\theta(X, \alpha, \beta) \tau^{\alpha}=\tau^{\beta} \theta(X, \alpha, \beta)$ (localized away from two). This proves

Proposition D12. If $\alpha, \beta \in \widetilde{K O}(X)=\tilde{K}_{\text {Diff }}(X)$ are stably fibre homotopy equivalent, there exists an equivariant isomorphism

$$
\theta(X, \alpha, \beta):\left(\varsigma^{\text {Diff }}(X)_{o d d}, \tau^{\alpha}\right) \rightarrow\left(\varsigma^{\text {Diff }}(X)_{o d d}, \tau^{\beta}\right)
$$

Now consider what happens when we localize at zero. If $\alpha, \beta \in \tilde{K}(X)$ and $\omega_{1}(\alpha)=\omega_{1}(\beta)$, then $\omega_{1}(\beta-\alpha)=0$. Hence using C17 in place of C11 and C15 we have:

Proposition D13. (a) If $\alpha, \beta \in \tilde{K}_{\text {Top }}(X)$ satisfy $\omega_{1}(\alpha)=\omega_{1}(\beta)$, then localized at zero, $\tau^{\alpha}=\tau^{\beta}$ on $\mathcal{S}^{\mathrm{Top}}(X)_{0}$.

(b) If $\alpha, \beta \in \tilde{K}_{\mathrm{Diff}}(X)$ satisfy $\omega_{1}(\alpha)=\omega_{1}(\beta)$, then localized at zero, $\theta(X, \alpha, \beta)$ : $\left(\mathcal{S}^{\text {Diff }}(X)_{(0)}, \tau^{\alpha}\right) \rightarrow\left(\mathcal{S}^{\text {Diff }}(X)_{(0)}, \tau^{\beta}\right)$ is an equivariant isomorphism.

Thus $\mathcal{S}$ is a GH functor with the WK property, an $\mathbb{Q}$ transfer, and a homotopy invariant $Q$ involution.

Proof of THeOrem A(2). Since the forgetful map $C^{\text {Diff }}(M) \rightarrow C^{\text {Top }}(M)$ commutes with transfer for $\xi: E \rightarrow M$ a Diff bundle, and involution (see remark following $\mathrm{C15}$ ), and $\gamma(M)$ defining the WK property, there is a natural transformation $F: \mathcal{S}^{\text {Diff }} \rightarrow \mathcal{S}^{\text {Top }}$ compatible with transfer, involution and the WK property. Consequently it induces the natural transformation $F_{ \pm}: \mathcal{S}_{ \pm}^{\text {Diff }} \rightarrow \mathcal{S}_{ \pm}^{\text {Top }}$.

(3) In [BLR] it is proved that $C \Re^{\text {Diff }}(M) \rightarrow C \Re^{\text {Top }}(M)$ is an equivalence.

(4) By $\mathrm{C} 12$ the fibre of $\mathcal{S}^{\text {Diff }}(X) \rightarrow \mathcal{S}^{\text {Top }}(X)$ is a homology theory, with coefficients $\hat{\mathcal{S}}^{\text {Diff }}(*)\left(\right.$ since $\left.\mathcal{S}^{\text {Top }}(*)=*\right)$.

To prove the result for $\mathcal{S}_{ \pm}(x, \alpha)$ we show that

$$
\pi_{i}\left(\operatorname{Fibre}\left(\mathcal{S}_{ \pm}^{\text {Diff }}(X, \alpha) \rightarrow \mathcal{S}_{ \pm}^{\text {Top }}(X, \alpha)\right)\right)
$$

does not depend on $\alpha$ :

As in $\$ \S \mathrm{B} 7-\mathrm{B} 9$, if $M^{n}=N^{n-1} \times I$, one may identify $C^{\text {Top/Diff }}(M)=$ $C^{\text {Top }}(M) / C^{\text {Diff }}(M)$ with Fibre $\left(B_{\#} C^{\text {Diff }}(M) \rightarrow B_{\#} C^{\text {Top }}(M)\right)$. If $\alpha$ is an orthogonal $2 k+1$ disc bundle over $M, T_{0}: C^{\text {Top/Diff }}(M)_{\text {odd }} \rightarrow C^{\text {Top } / \text { Diff }}\left(E(\alpha)_{0}\right)_{\text {odd }}$ is an $\omega(n)$ isomorphism, $k$ sufficiently large, by the Mayer-Vietoris sequence $\mathrm{C} 13$ and the argument in $\mathrm{C} 14$. By the argument of $\mathrm{C} 15$ this leads to an $\omega(n)$ isomorphism $\theta(\alpha)$ : $C^{\text {Top/Diff }}(M)_{\text {odd }} \rightarrow C^{\text {Top/Diff }}(E(\alpha))_{\text {odd }}$ for $\alpha$ of any dimension, such that $\theta(\alpha)$ commutes with $\tau$ up to the appropriate sign. Applying this to $\left(N^{n}(x), r_{n}^{*} \alpha\right)$, the result follows.

(5) Let $M^{n}$ be an $氏$ manifold and $\alpha=T M$. Then $N^{n+k}(M)=E\left(\nu^{k}\right), \nu^{k}$ the normal $k$ disc bundle in $E^{n+k}, k$ large. Now

$$
\hat{\tau}_{n+k}^{\alpha}=(-1)^{n+k+n} T\left(r_{n+k}^{*} \alpha\right)^{-1} \circ \tau_{n+k}^{\alpha} \circ T\left(r_{n+k}^{*} \alpha\right) .
$$


But $\nu_{k} \circ r_{n+k}^{*} \alpha=1_{n+k}: M \times D^{n+k} \rightarrow M$. So in the stable range $\tau_{n+k}^{\alpha} \Sigma^{n+k}=$ $(-1)^{n+k} \Sigma^{n+k} \tau_{M}, \tau_{M}$ the involution on $C(M)$. Hence

$$
\begin{aligned}
\hat{\tau}_{n+k}^{\alpha} & =(-1)^{n} T\left(r_{n+k}^{*} \alpha\right)^{-1} \circ \Sigma^{n+k} \tau_{M} \circ\left(\Sigma^{n+k}\right)^{-1} \circ T\left(r_{n+k}^{*} \alpha\right) \\
& =(-1)^{n} T\left(\nu^{k}\right) \circ \tau_{M} \circ T\left(\nu^{k}\right)^{-1} .
\end{aligned}
$$

Let $\theta \simeq \lim _{\vec{k}} T\left(\nu^{k}\right), T\left(\nu^{k}\right): \tilde{C} / C(M) \rightarrow \tilde{C} / C\left(N^{n+k}(M)\right)$; then:

(a) $\theta: \overrightarrow{\tilde{C}} / C(M) \rightarrow \delta(M)$ is an $\omega(n)$ equivalence, and $\tau^{\alpha}=(-1)^{n} \theta \cdot \tau_{M}$.

By C3, $\pi_{i} \tilde{C} / C(M) \rightarrow \pi_{i} \tilde{A} / A(M)$ is $(\omega(n+1)+1)$-connected; hence

(b) $\tilde{A} / A(M)_{\text {odd }} \stackrel{\omega(n)}{\sim} \delta_{e(n)}(M)$.

(c) The result $C \Re(M) \stackrel{\omega(n)}{\sim} \Omega$ Nil $\delta(H)$ follows from (a) above and the remark at the end of the section on the WK property.

(d) The result $\mathscr{T}(M)_{\text {odd }} \stackrel{\omega(n)}{\sim}$ Nil $\delta_{e(n)}(M)$ follows from (b) and the results of $\left[\mathbf{B}_{2}\right]$.

Proof of Theorem B. From the definition (proof of C7(b)) of $\beta=\beta(M)$ : $[\tilde{\mathcal{H}}(M)]_{\omega(n)+1} \rightarrow[\tilde{C} / C(M)]_{\omega(n)+1}$ we see that on the $\omega(n)$ Postnikov term

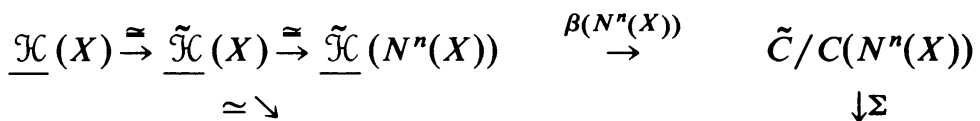

$$
\begin{aligned}
& \tilde{\tilde{\mathcal{H}}}\left(N^{n+1}(X)\right) \quad \stackrel{\beta\left(N^{n+1}(X)\right)}{\rightarrow} \tilde{C} / C\left(N^{n+1}(X)\right)
\end{aligned}
$$

commutes, up to homotopy. Hence we can define $\beta: \mathcal{H}(X) \rightarrow \delta(X)$.

The other results in Theorem $B$ then follow trivially from the definition.

Proof OF THEOREM C. This follows from C7.

Proof of Corollary D. This follows from C8 and Theorem A5.

Proof of Corollary E. Given $p: M \rightarrow \partial M$ with $M \stackrel{p}{\rightarrow} \partial M \stackrel{i}{\rightarrow} M$ homotopic to $1_{M}$, then the inclusion $\tilde{A}(M) \rightarrow \tilde{\mathcal{H}}(M)$ which factors through $\tilde{\mathcal{H}}(M)$ is trivial. In fact, $\tilde{\mathcal{H}}(M) \rightarrow \tilde{\mathcal{H}}(M)$ is homotopically trivial since if $f \in \tilde{\mathcal{H}}(M)$, then in $\tilde{\mathcal{H}}(M)$, $f=f \circ 1_{M} \sim \overline{f \circ} i \circ p \sim i \circ p \sim 1_{M}$. The result follows from C 7 .

Proof of Corollary F. (1) Given $M^{n} \subset E^{n}$, we can choose $n$ discs $D_{a}^{n} \subset M^{n}$ $\subset D_{b}^{n}$. By Proposition B1, $\tilde{A} / A\left(D_{a}^{n}\right) \rightarrow \tilde{A} / A\left(M^{n}\right)$ is $(2 r-2)$ - (resp. $\inf (2 r-2, r+3)-)$ connected in the Diff (resp. Top) category. Since

$$
\tilde{A} / A\left(D_{a}^{n}\right) \rightarrow \tilde{A} / A\left(M^{n}\right) \rightarrow \tilde{A} / A\left(D_{b}^{n}\right)
$$

is an isomorphism (essentially the identity), $\tilde{A} / A\left(M^{n}\right) \rightarrow \tilde{A} / A\left(D_{b}^{n}\right)$ is a $2 r-2$ (resp. $\inf (2 r-2, r+3)$ ) equivalence. Localized away from two and in the stable range we have the commutative diagram

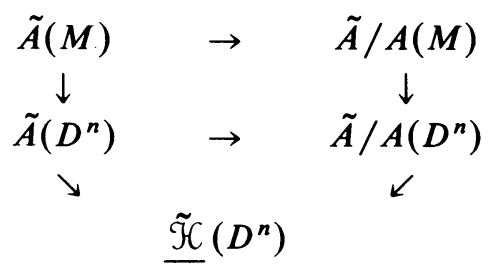

Since $\tilde{\mathcal{H} C}\left(D^{n}\right)$ is contractible, $q: \tilde{A}(M) \rightarrow \tilde{A} / A(M)$ is trivial on $\left[\tilde{A}(M)_{\text {odd }}\right]_{k}, k \leqslant$ $\inf \left(\omega(n)^{\text {Diff }}, 2 r-2\right)\left(\right.$ resp. $\left.k \leqslant \inf \left(\omega(n)^{\text {Top }}, 2 r-2, r+3\right)\right)$. The result follows. 
(2) Consider the map of fibrations

$$
\begin{array}{ccccc}
\tilde{A}\left(M \times S^{1} ; M\right)_{0} & \rightarrow & \tilde{A}\left(M \times S^{1}\right)_{0} & \stackrel{r}{\rightarrow} & \tilde{E}\left(M, M \times S^{1}\right)_{0} \\
\downarrow i & & \downarrow i & & \downarrow i \\
\tilde{\tilde{H}}\left(M \times S^{1} ; M\right)_{0} & \rightarrow & \underline{\tilde{H}}\left(M \times S^{1}\right)_{0} & \stackrel{r}{\rightarrow} & \operatorname{Maps}\left(M, M \times S^{1}\right)_{0}
\end{array}
$$

where ()$_{0}$ denotes the component of the identity or the inclusion. It is easy to check that we have product decompositions:

$$
\underline{\tilde{\mathcal{H}}}\left(M \times S^{1}\right)_{0}=\underline{\tilde{\mathcal{K}}}\left(M \times S^{1}\right)_{1} \times S^{1}, \quad \operatorname{Maps}\left(M, M \times S^{1}\right)_{0}=\underline{\tilde{\mathcal{K}}}(M)_{0} \times S^{1},
$$

and if $\partial M=\varnothing$,

$$
\tilde{A}\left(M \times S^{1}\right)_{0}=\tilde{A}\left(M \times S^{1}\right)_{1} \times S^{1}, \quad \tilde{E}\left(M, M \times S^{1}\right)_{0}=\tilde{E}\left(M, M \times S^{1}\right)_{1} \times S^{1},
$$

where ()$_{1}$ is the subspace of ()$_{0}$, sending (*), or $(*, *)$ into $M \times(*)$. (If $\partial M \neq \varnothing$, $\tilde{A}\left(M \times S^{1}\right)_{0}=\tilde{A}\left(M \times S^{1}\right)_{1}$ and $\tilde{E}\left(M, M \times S^{1}\right)_{0}=\tilde{E}\left(M, M \times S^{1}\right)_{1}$.) The map of fibrations respects these decompositions (up to homotopy).

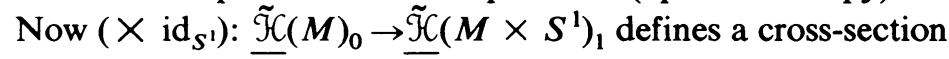

$$
s: \operatorname{Maps}\left(M, M \times S^{1}\right)_{0} \rightarrow \tilde{\mathscr{H}}\left(M \times S^{1}\right)_{0} \text {. }
$$

Also according to $\left[\mathbf{B}_{2}\right]$, the fibre of $\lambda: \tilde{E}\left(M, M \times S^{1}\right)_{1} \rightarrow \tilde{E}(M, M \times R)_{1} \sim$ $\tilde{E}(M, M \times R)_{0}$ (the lift to the covering space) becomes trivial when localized away from two. Further $r: \tilde{A}(M \times R)_{0} \rightarrow \tilde{E}(M, M \times R)_{0}$ has trivial fibre, and $\lambda$ : $\tilde{A}\left(M \times S^{1}\right)_{1} \rightarrow \tilde{A}(M \times R)_{1} \sim \tilde{A}(M \times R)_{0}$ has a section $j: \tilde{A}(M \times R)_{1} \rightarrow$ $\tilde{A}\left(M \times S^{1}\right)_{1}\left[\right.$ BLR]. Thus $j r^{-1} \lambda: \tilde{E}\left(M, M \times S^{1}\right)_{1} \rightarrow \tilde{A}\left(M \times S^{1}\right)_{1}$ defines a map $s=j r^{-1} \lambda \times \mathrm{id}_{S^{1}}$ which when localized away from two becomes a cross-section of $r: \tilde{A}\left(M \times S^{1}\right)_{0} \rightarrow \tilde{E}\left(M, M \times S^{1}\right)_{0}$ such that

$$
\begin{array}{ccc}
\tilde{A}\left(M \times S^{1}\right)_{0} & \stackrel{s}{\leftarrow} & \tilde{E}\left(M, M \times S^{1}\right)_{0} \\
\downarrow i & & \downarrow i \\
\tilde{\tilde{\mathcal{C}}}\left(M \times S^{1}\right)_{0} & \stackrel{s}{\leftarrow} & \operatorname{Maps}\left(M, M \times S^{1}\right)_{0}
\end{array}
$$

commutes up to homotopy. By the remark following the proof of $\mathrm{C} 13$, on the $\omega(n)+1$ Postnikov term:

$$
\begin{array}{ccr}
\tilde{\mathcal{F}}(M)_{0} & \stackrel{\beta(M)}{\rightarrow} & \tilde{C} / C(M) \\
\left(\times \mathrm{id}_{S^{1}}\right) \downarrow & & \downarrow\left(\times \mathrm{id}_{S^{\prime}}\right) \\
\underline{\mathcal{H}}\left(M \times S^{1}\right)_{0} & \stackrel{\beta\left(M \times S^{1}\right)}{\rightarrow} & \tilde{C} / C\left(M \times S^{1}\right)
\end{array}
$$

commutes. Since

$$
\left(\times \mathrm{id}_{S^{1}}\right): C(M) \rightarrow C\left(M \times S^{1}\right)
$$

is trivial [BLR], $\beta\left(M \times S^{1}\right) \circ\left(\times \mathrm{id}_{S^{\prime}}\right)$ and hence $\beta \circ i \circ s: \tilde{E}\left(M, M \times S^{1}\right)_{0} \rightarrow$ $\tilde{C} / C\left(M \times S^{1}\right)$ is trivial.

On the other hand

$$
\begin{array}{ccc}
\tilde{A}(M \times I)_{0} & \tilde{\rightarrow} & \tilde{A}\left(M \times S^{1} ; M\right)_{0} \\
i \downarrow & & i \downarrow \\
\tilde{\mathcal{H}}(M \times I ; M \times \partial I)_{0} & \rightarrow & \underline{\tilde{\mathcal{K}}}\left(M \times S^{1} ; M\right)_{0}
\end{array},
$$


induced by embedding $I$ in $S^{1}$ [BLR] commutes, with the top row an equivalence [BLR]. But $\tilde{\mathcal{H}}(M \times I ; M \times \partial I) \rightarrow \tilde{\mathcal{H}}\left(M \times S^{1} ; M\right)_{0}$ is trivial since it factors through the contractible space $\tilde{\mathcal{F} C}(M \overline{\times} I ; M \times 0)$.

Thus when localized away from two, $\beta \circ i: \tilde{A}\left(M \times S^{1}\right)_{0} \rightarrow \tilde{C} / C(M)$ is trivial on the first two factors of

$$
\left(\tilde{A}\left(M \times S^{1}\right)_{0}\right)_{\text {odd }} \sim\left(\tilde{A}\left(M \times S^{1} ; M\right)_{0}\right)_{\text {odd }} \times\left(\tilde{E}\left(M, M \times S^{1}\right)_{0}\right)_{\text {odd }} \times S_{\text {odd }}^{1}
$$

Thus $\Omega(\beta \circ i):\left(\Omega \tilde{A}\left(M \times S^{1}\right)\right)_{\text {odd }} \rightarrow(\Omega \tilde{C} / C(M))_{\text {odd }}$ is trivial on the $\omega(n)$ Postnikov term (by $\Omega X$ we mean the loop space of the 1-connected cover of $X$ ). The result follows.

(3) Let $M$ be a compact $K(\Pi, 1)$ manifold. Then

$$
\pi_{i}\left(\underline{\tilde{\mathcal{K}}}(M)_{0}\right) \cong \pi_{0}\left(\operatorname{Maps}\left(S^{i} \times M, M ;(*) \times M\right)\right) \simeq \begin{cases}0, & \text { if } i \neq 1, \\ \Pi, & \text { if } i=1,\end{cases}
$$

i.e. $\underline{\tilde{\mathcal{H}}}(M)_{0} \sim M$. Thus $\Omega(\underline{\tilde{\mathcal{H}}}(M))$ is contractible. The result follows.

Appendix. Proof of $\Sigma \sim \phi$. Let $h \in C(M)$. In the diagrams below the rectangles represent $M \times I^{2}$. All homotopies are fixed on $M \times(0 \times I \cup I \times \partial I)$. The arrows represent the direction from 0 to 1 , and the arcs represent rotation of $h$ or $h^{-1}$ by $90^{\circ}$ as indicated. We first note (" $\circ "$ means composition of indicated isomorphisms)

(a)
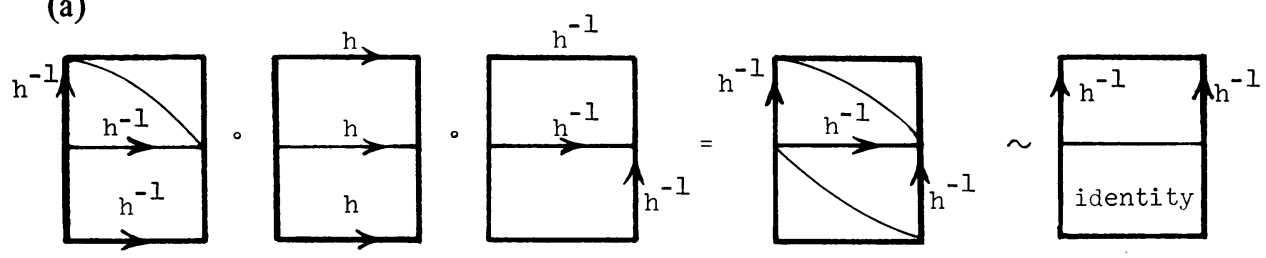

(b)
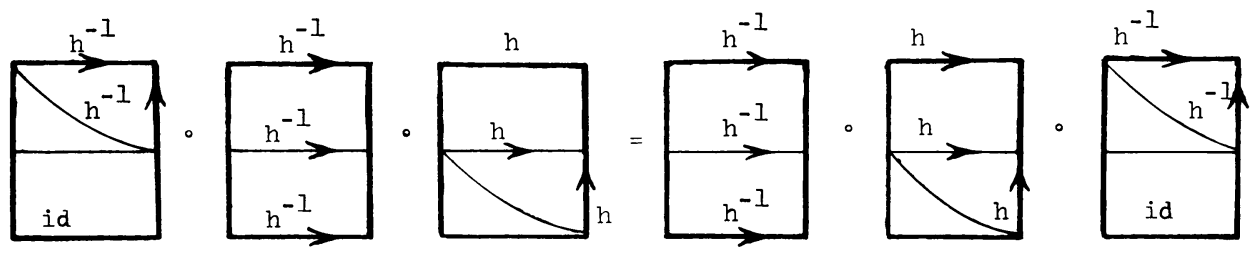

ProOF.

$\Sigma(\mathrm{h})=$
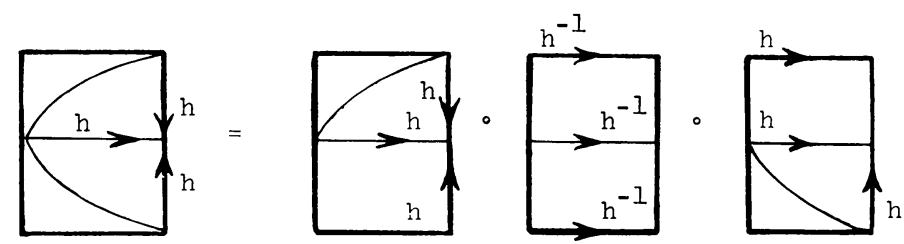
Now insert the inverse of the right side of (a) composed with the left side of (a), between the first and second diagrams in the above composition. Since

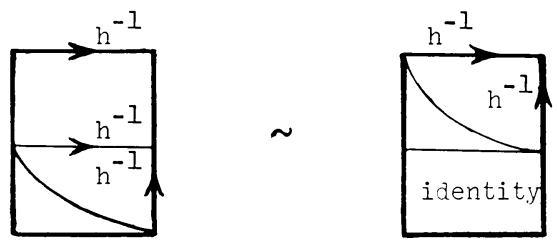

it follows from (b) that $\Sigma(h) \sim$
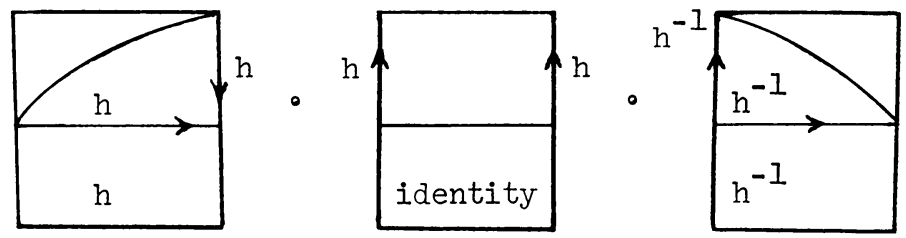

$\sim \phi(h)$

\section{REFERENCES}

[ABK] P. Antonelli, D. Burghelea and P. Kahn, Concordance homotopy groups of geometric automorphism groups, Lecture Notes in Math., vol. 215, Springer-Verlag, Berlin and New York, 1971.

[B] D. Burghelea, Automorphisms of manifolds, Proc. Sympos. Pure Math., vol. 32, Amer. Math. Soc., Providence, R. I., 1978, pp. 347-371.

$\left[\mathrm{B}_{2}\right] \ldots$, The structure of block automorphisms of $M \times S^{1}$, Topology 16 (1977), 67-78.

$\left[\mathbf{B}_{3}\right]$, The rational homotopy groups of $\operatorname{Diff}(M)$ and $\operatorname{Homeo}(M)$ in the stability range, Proc. Conf. Algebraic Topology (Aarhus, 1978), Lecture Notes in Math., vol. 763, Springer, Berlin and New York, pp. 604-626.

[BL] D. Burghelea and R. Lashof, Stability of concordances and suspension homeomorphism, Ann. of Math. 15 (1977), 449-472.

[BLR] D. Burghelea, R. Lashof and M. Rothenberg, Groups of automorphisms of compact manifolds, Lecture Notes in Math., vol. 473, Springer-Verlag, Berlin and New York, 1975.

[H] A. Hatcher, Higher simple homotopy theory, Ann. of Math. 102 (1975), 101-137.

$\left[\mathbf{H}_{2}\right]$ , Concordance spaces, Proc. Sympos. Pure Math., vol. 32, Amer. Math. Soc., Providence, R. I., 1978.

[HJ] W. C. Hsiang and B. Jahren, On the homotopy groups of the diffeomorphism groups of spherical space forms (preprint).

[L] R. Lashof, Embedding spaces, Illinois J. Math. 78 (1976), 144-154.

[M] P. May, The geometry of iterated loop spaces, Lecture Notes in Math., vol. 271, Springer-Verlag, Berlin and New York, 1972.

[W] F. Waldhausen, Algebraic k-theory of topological spaces. I, Proc. Sympos. Pure Math., vol. 32, Amer. Math. Soc., Providence, R. I., 1978, pp. 35-60.

Department of Mathematics, Ohio State University, Columbus, Oho 43210

Department of Mathematics, University of Chicago, Chicago, Illinois 60637 